\title{
Synaptic Vesicle Populations in Saccular Hair Cells Reconstructed by Electron Tomography
}

\author{
David Lenzi, ${ }^{1}$ Jonathan W. Runyeon, ${ }^{1}$ John Crum, ${ }^{2}$ Mark H. Ellisman, ${ }^{2}$ and William M. Roberts ${ }^{1}$ \\ 1/nstitute of Neuroscience, University of Oregon, Eugene, Oregon 97403-1254, and ${ }^{2}$ National Center for Microscopy and \\ Imaging Research and Department of Neurosciences, University of California, San Diego, La Jolla, California 92093-0608
}

\begin{abstract}
We used electron tomography to map the three-dimensional architecture of the ribbon-class afferent synapses in frog saccular hair cells. The synaptic body (SB) at each synapse was nearly spherical (468 $\pm 65 \mathrm{~nm}$ diameter; mean $\pm \mathrm{SD}$ ) and was covered by a monolayer of synaptic vesicles (34.3 nm diameter; $8.8 \%$ coefficient of variation), many of them tethered to it by $\sim 20$-nm-long filaments, at an average density of $55 \%$ of closepacked (376 \pm 133 vesicles per SB). These vesicles could support $\sim 900$ msec of exocytosis at the reported maximal rate, which the cells can sustain for at least $2 \mathrm{sec}$, suggesting that replenishment of vesicles on the SB is not rate limiting. Consistent with this interpretation, prolonged $\mathrm{K}^{+}$depolarization did not deplete vesicles on the SB. The monolayer of SBassociated vesicles remained after cell lysis in the presence of $4 \mathrm{~mm} \mathrm{Ca}^{2+}$, indicating that the association is tight and $\mathrm{Ca}^{2+}$ resistant. The space between the SB and the plasma mem-
\end{abstract}

brane contained numerous vesicles, many of which $(\sim 32$ per synapse) were in contact with the plasma membrane. This number of docked vesicles could support maximal exocytosis for at most $\sim 70 \mathrm{msec}$. Additional docked vesicles were seen within a few hundred nanometers of the synapse and occasionally at greater distances. The presence of omega profiles on the plasma membrane around active zones, in the same locations as coated pits and coated vesicles labeled with an extracellular marker, suggests that local membrane recycling may contribute to the three- to 14-fold greater abundance of vesicles in the cytoplasm (not associated with the SB) near synapses than in nonsynaptic regions.

Key words: active zone; docked vesicles; endocytosis; exocytosis; hair cell; microperoxidase; Rana pipiens; ribbon synapse; sacculus; synaptic body; synaptic vesicle; tomography; vesicle concentration
Experiments that use neurons and neurosecretory cells have revealed multiple kinetic components of exocytosis that may correspond to "pools" of vesicles in different physiological states (Neher, 1998). Some of these pools may be distinguishable morphologically (e.g., vesicles in contact with the plasma membrane or bound to intracellular structures). Understanding the correspondence between vesicle pools defined on physiological and morphological grounds and how vesicles move between these pools remains a challenge that will require a combination of physiology and quantitative ultrastructural analysis.

The ribbon-class synapses in sensory receptors and neurons in the ear and retina are well suited for this level of analysis. The favorable electrical properties of these cells have allowed the use of capacitance measurements to study the kinetics of synaptic exocytosis in response to step changes in membrane potential (Parsons et al., 1994; Rieke and Schwartz, 1994; von Gersdorff and Matthews, 1994; Mennerick and Matthews, 1996; von Gersdorff et al., 1996) and $\mathrm{Ca}^{2+}$ (Heidelberger et al., 1994; Rieke and Schwartz, 1996). On the basis of these physiological experiments

\footnotetext{
Received May 26, 1998; revised Sept. 10, 1998; accepted Oct. 12, 1998.

This work was funded by National Institutes of Health Grant NS27142 to W.M.R., United States Public Health Service Grants RR04050 and NS36069 to M.H.E., and a Deafness Research Foundation grant to D.L. D.L. was supported in part by postdoctoral fellowships from the Muscular Dystrophy Association and the Natural Sciences and Engineering Research Council of Canada. We thank C. E. Armstrong and B. Edmonds for helpful comments on this manuscript, Eric Schabtach and Naoko Yamada for help with electron microscopy, and Martin Hadida-Hassan and the three Steves-Lamont, Peltier, and Young - for help with programming and telemicroscopy.

Correspondence should be addressed to Dr. William M. Roberts, Institute of Neuroscience, University of Oregon, Eugene, OR 94703-1254.

Copyright (C) 1998 Society for Neuroscience $\quad 0270-6474 / 98 / 190119-14 \$ 05.00 / 0$
}

and recent ultrastructural data (von Gersdorff et al., 1996), it has been proposed that the small population of vesicles docked at the membrane beneath the ribbons is responsible for the most rapid phase of exocytosis (the "ultrafast pool"; Mennerick and Matthews, 1996), whereas the remaining vesicles tethered to the ribbons are available more slowly (the "rapidly released pool"; von Gersdorff et al., 1996). Once these two pools are exhausted, exocytosis slows markedly or ceases (von Gersdorff and Matthews, 1994). In frog saccular hair cells (Parsons et al., 1994), exocytosis can be maintained continuously for 10 times as long as that reported for bipolar cells (von Gersdorff et al., 1996) (but see Lagnado et al., 1996), suggesting functional differences between these two ribbon-class synapses. The synapses also differ in ultrastructure. The ribbons in bipolar terminals are flat ellipsoids, whereas the corresponding synaptic body (SB) in frog saccular hair cells is spherical.

In the present study we use electron tomography to examine the three-dimensional ultrastructure of afferent junctions in frog saccular hair cells and interpret these results in light of previous physiological data from these cells. By using images of a single thick section taken from many different angles, electron tomography provides a convenient means of obtaining information equivalent to a stack of thin sections through the tissue without the need for cutting serial sections. Electron tomography captures large portions of synaptic substructure in each reconstruction, eliminates the problem of lost sections, and provides $z$-axis resolution that is finer than the thickness of a thin section. We present quantitative data about several morphologically distinct pools of vesicles in these cells, including the demonstration that numerous morphologically docked vesicles are present beneath 
each SB. This information, plus evidence that vesicles are bound tightly to the SB and that endocytosis occurs locally at synapses, provides constraints on functional models that can be tested in future physiological and anatomical studies of this synapse. This report is the first use of electron tomography to examine the detailed ultrastructure of a synapse.

\section{MATERIALS AND METHODS}

\section{Transmission electron microscopy}

We studied hair cells from the sacculus of the frog, Rana pipiens. The basic protocol was to remove the sacculus from the inner ear cavity and then dissect the saccular macula free of the otolith in normal physiological saline containing (in $\mathrm{mm}$ ): $110 \mathrm{Na}^{+}, 2 \mathrm{~K}^{+}, 4 \mathrm{Ca}^{2+}, 118 \mathrm{Cl}^{-}, 5$ HEPES, pH 7.25, and 3 D-glucose (Roberts et al., 1990). The tissue was fixed for $2 \mathrm{hr}$ in $3 \%$ glutaraldehyde buffered with $100 \mathrm{~mm}$ Na-cacodylate, $\mathrm{pH}$ 7.3. To prevent the calcified otoconia from damaging the microtome knife, we decalcified sacculi for $3 \mathrm{hr}$ in buffer supplemented with $35 \mathrm{~mm}$ EDTA and $4 \mathrm{~mm} \mathrm{CaCl}_{2}$ (Roberts et al., 1990); then the sacculi were rinsed in buffer, post-fixed for $1 \mathrm{hr}$ in $2 \%$ aqueous $\mathrm{OsO}_{4}$ at $4^{\circ} \mathrm{C}$, rinsed in $\mathrm{H}_{2} \mathrm{O}$, and stained in $5 \%$ aqueous uranyl acetate for $4 \mathrm{hr}$ at $60^{\circ} \mathrm{C}$. The fixed tissue was dehydrated via an ethanol series into acetone and then transferred into resin (Epon or Araldite) before polymerization at $60^{\circ} \mathrm{C}$. Heavy metals, Na-cacodylate buffer, fixative, and embedding media were obtained from Electron Microscopy Sciences (Fort Washington, PA). Other salts and chemicals were obtained from Sigma (St. Louis, MO).

Conventional transmission electron microscopy (TEM). To visualize coated pits, we depolarized hair cells for $30 \mathrm{~min}$ at $4^{\circ} \mathrm{C}$ in $22 \mathrm{~mm} \mathrm{~K}+$ saline containing (in mM): $90 \mathrm{Na}^{+}, 22 \mathrm{~K}^{+}, 4 \mathrm{Ca}^{2+}, 118 \mathrm{Cl}^{-}, 5 \mathrm{HEPES}$, $\mathrm{pH} 7.25$, and $3 \mathrm{D}$-glucose. In other experiments the hair cells were depolarized for up to $30 \mathrm{~min}$ at room temperature in 45 or $80 \mathrm{~mm} \mathrm{~K}^{+}$ saline (same as above, but with $\mathrm{K}^{+}$substituted for $\mathrm{Na}^{+}$). Then the cells were fixed and processed as in the basic protocol. For microperoxidase uptake experiments, the sacculi were dissected and incubated for $90 \mathrm{~min}$ in zero calcium saline (normal saline, but with $\mathrm{NaCl}$ substituted for $\mathrm{CaCl}_{2}$ ). The otolithic membrane then was removed, and a furrow was plowed across the sensory epithelium (Armstrong and Roberts, 1998) to provide access to the basolateral surfaces of hair cells unimpeded by the tight junctions of the intact epithelium. Cells were exposed to microperoxidase $(4.8 \% \mathrm{w} / \mathrm{v})$ for $15 \mathrm{~min}$ in normal saline, lightly fixed for $10 \mathrm{~min}$

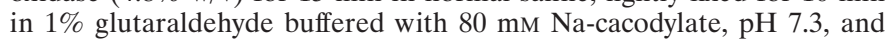
rinsed overnight in buffer. Then the tissue was preincubated in $0.5 \%$ $(\mathrm{w} / \mathrm{v})$ diaminobenzidine (DAB) in $100 \mathrm{~mm}$ Tris buffer, $\mathrm{pH}$ 7.25, for $1 \mathrm{hr}$, reacted in DAB solution supplemented with $0.01 \% \mathrm{H}_{2} \mathrm{O}_{2}$ for $2 \mathrm{hr}$ in the dark, rinsed in Tris buffer that was followed by Na-cacodylate buffer, fixed in $3 \%$ glutaraldehyde, decalcified, post-fixed, stained, and embedded as in the basic protocol.

Gray $(40-50 \mathrm{~nm})$ or silver-gray $(50-60 \mathrm{~nm})$ sections were cut with a glass or diamond knife, collected onto Butvar-coated grids, and stained for $25 \mathrm{~min}$ at $40^{\circ} \mathrm{C}$ in $5 \%$ aqueous uranyl acetate and for $2 \mathrm{~min}$ in lead citrate before being rinsed in $\mathrm{H}_{2} \mathrm{O}$ and carbon-coated. Sections were imaged at $80 \mathrm{kV}$ by a Philips CM-12 electron microscope (Mahwah, NJ).

Electron tomography. We used two procedures to stain tissue for tomographic reconstruction. Osmium was used to stain the SB and vesicles, whereas ethanolic phosphotungstic acid (EPTA) was used to enhance the staining of the presynaptic plasma membrane and provide SBs that were less electron-opaque. For osmium-stained material we followed the basic protocol. For EPTA-stained material we dissected sacculi in low calcium saline (zero calcium saline with $50 \mu \mathrm{M} \mathrm{CaCl}_{2}$ added) and fixed them for $1 \mathrm{hr}$ in $2 \%$ glutaraldehyde in buffer containing $80 \mathrm{~mm}$ Na-cacodylate, $\mathrm{pH} 7.3$, and $4 \mathrm{mM} \mathrm{CaCl}_{2}$. Saccular maculae were rinsed in buffer, decalcified in buffered EDTA, rinsed, and dehydrated via an ethanol series into 95\% EtOH. Then they were stained for $1 \mathrm{hr}$ in $1 \%$ phosphotungstic acid in $100 \%$ EtOH containing two drops of $95 \%$ EtOH per $10 \mathrm{ml}$ of solution (Fry and Spira, 1980), transferred to $100 \%$ $\mathrm{EtOH}$, and embedded in resin.

Thick sections $(0.25,0.5$, or $1.0 \mu \mathrm{m})$ were collected onto clamshell grids and stained for $10 \mathrm{~min}$ in $1 \%$ aqueous uranyl acetate, followed by $1 \mathrm{~min}$ in lead salts (Sato, 1967). After being washed in $\mathrm{ddH}_{2} \mathrm{O}, 10 \mathrm{~nm}$ colloidal gold particles were deposited on one surface of the section, and both surfaces were carbon-coated. Synapses were imaged at a magnification of $25,000-60,000 \times$ by a JEOL $4000 \mathrm{EX}$ intermediate voltage electron microscope (IVEM) that was operated at an accelerating voltage of $400 \mathrm{kV}$. The section was oriented with the $x-y$ plane perpendicular to the electron beam, and a series of 61 or 121 micrographs was acquired on film by rotating the specimen $\pm 60^{\circ}$ around the $y$-axis in $1^{\circ}$ or $2^{\circ}$ tilt increments with a computer-controlled goniometer. To minimize shrinkage of the section thickness during the tilt series and reduce the effects of within-tilt-series variation on the subsequent backprojection, we preirradiated sections for $15 \mathrm{sec}$ at each tilt angle before image acquisition. Preirradiating the section in this way subjected the specimen to the steepest portion of the nonlinear shrinkage curve before data were collected (Luther, 1992).

Telemicroscopy. Some data sets were acquired via a telemicroscopy system developed by the National Center for Microscopy and Imaging Research (Young et al., 1996), which allowed us to remotely control the IVEM in California from our laboratory at the University of Oregon via the internet. Before a telemicroscopy session a survey of the grid was obtained as a mosaic of low-magnification $(3000 \times)$ digital images. The survey was transferred to our laboratory in Oregon, annotated to highlight regions of interest with Adobe Photoshop (Mountain View, CA), and sent back to San Diego. This survey was used as a guide during the interactive session in which we used a graphical interface, GridManager, to control the microscope and examine the specimen at high magnification. Once we had identified a region to image for tomography, the interactive session was terminated and the microscopist in San Diego acquired the tilt series.

\section{Tomography}

Image processing was performed with the SUPRIM software suite (Schroeter and Bretaudiere, 1996). Each negative of the tilt series was digitized with a 14-bit cooled CCD camera. The $1024 \times 1024$ pixel images were aligned by using the colloidal gold particles as fiducial marks that could be tracked over a range or all of the tilt series. At least eight marks on each negative were selected manually using the program FIDO (Soto et al., 1994). Then the selected fiducial marks were input to the program SAXALIGN (Jing and Sachs, 1991), which used a leastsquared-error algorithm to solve for the translation and rotation required to align each image in the tilt series to a common tilt axis. The quality of the alignment was checked with FICHECK (Soto et al., 1994), and the alignment was repeated if necessary after the specification of the fiducial marks was corrected with FIDO. The three-dimensional volume of the tissue section was reconstructed by $r$-weighted backprojection (Radermacher, 1992) with the use of SUPRIM, producing a volume up to $600 \times$ $600 \times 351$ voxels, which were $1.3-5.5 \mathrm{~nm}$ cubes. The ultrastructure of the synapse then could be inspected throughout the volume by shuffling through adjacent slices, one voxel thick, along the $x$-, $y$-, or $z$-axis. Because resolution is poorest in the $z$-dimension (Radermacher, 1992), we viewed the data in the $x-y$ plane, moving through the volume along the $z$-axis. Organelles and structures were identified and traced manually in each plane with XVOXTRACE before they were rendered with the SYNU set of programs (Hessler et al., 1992).

\section{Geometrical analysis}

To determine the center and diameter of a synaptic vesicle, we identified the $x-y$ plane of largest cross section, traced the perimeter of the vesicle along the middle of the membrane bilayer, and fit a circle of equal area to the traced polygon (Bashein and Detmer, 1994). Then the vesicle could be rendered as a sphere having the same center and diameter as the fitted circle. The distribution of vesicle diameters obtained from these fits was characterized by the mean and coefficient of variation $(C V$; the $\mathrm{SD}$ expressed as a fraction of the mean). To correct for the contribution of tracing errors to the $C V$, we determined the tracing variance and subtracted it from the total variance before calculating the $C V$. Vesicle volume was measured by tracing the vesicle in all planes in which it appeared, multiplying the area of each traced polygon by the plane thickness (the voxel size), and summing the resulting volumes. When only the vesicle diameters were measured, the $C V$ of vesicle volumes $\left(C V_{\text {vol }}\right)$ was estimated from the $C V$ of vesicle diameters $\left(C V_{\text {diam }}\right)$ by using the theoretical relationship:

$$
C V_{\text {vol }}=3 C V_{\text {diam }}\left[1+4 C V_{\text {diam }^{2}}^{2}+(5 / 3) C V_{\text {diam }^{4}}^{4}\right]^{1 / 2} /\left(1+3 C V_{\text {diam }^{2}}\right),
$$

which assumes that the diameter distribution is Gaussian and that the volume is proportional to the diameter cubed. This formula is well approximated by $C V_{\text {vol }}=3 C V_{\text {diam }}$ for the small $C V_{\text {diam }}$ values that we measured.

A vesicle was classified as SB-associated if, in the plane of its largest cross section, it was tethered to or within $30 \mathrm{~nm}$ of the SB. A vesicle was 
Table 1. Dimensions and vesicle concentrations at reconstructed synapses

\begin{tabular}{|c|c|c|c|c|c|c|c|c|c|c|}
\hline \multirow[b]{2}{*}{ Synapse } & \multirow[b]{2}{*}{ Stain } & \multicolumn{2}{|c|}{ Dimensions } & \multicolumn{5}{|c|}{ Synaptic body } & \multicolumn{2}{|c|}{ Outlying vesicles } \\
\hline & & $\begin{array}{l}\text { Voxel } \\
\text { Edge } \\
(\mathrm{nm})\end{array}$ & $\begin{array}{l}\text { Reconstructed } \\
\text { volume }(\mathrm{nm} \times \\
\mathrm{nm} \times \mathrm{nm})\end{array}$ & $\begin{array}{l}\text { Diameter } \\
(\mathrm{nm})\end{array}$ & $\begin{array}{l}\text { Portion re- } \\
\text { constructed } \\
(\%)\end{array}$ & $\begin{array}{l}\text { Vesicles } \\
\text { counted } \\
(\#) \\
\end{array}$ & $\begin{array}{l}\text { Total vesi- } \\
\text { cles/SB } \\
(\#)\end{array}$ & $\begin{array}{l}\text { Packing } \\
\text { density } \\
(\%)\end{array}$ & $\begin{array}{l}\text { Count } \\
(\#)\end{array}$ & $\begin{array}{l}\text { Packing } \\
\text { density } \\
(\%)\end{array}$ \\
\hline 1 & Os & 3.3 & $845 \times 845 \times 200$ & 524 & 29 & 162 & 562 & 68 & 164 & 6.5 \\
\hline 2 & Os & 2.1 & $840 \times 840 \times 215$ & 516 & 36 & 171 & 474 & 59 & 78 & 4.2 \\
\hline 3 & Os & 3.0 & $900 \times 900 \times 205$ & 362 & 34 & 89 & 265 & 60 & 67 & 4.0 \\
\hline \multirow[t]{2}{*}{4} & EPTA & 2.6 & $1470 \times 1125 \times 325$ & 581 & 47 & 231 & 494 & 50 & 287 & 3.9 \\
\hline & & & & 457 & 45 & 134 & 298 & 46 & & \\
\hline 5 & EPTA & 2.9 & $1145 \times 1145 \times 380$ & 477 & 75 & 139 & 186 & 26 & 208 & 3.4 \\
\hline 6 & EPTA & 1.3 & $770 \times 770 \times 390$ & 425 & 70 & 204 & 290 & 50 & 206 & 8.1 \\
\hline 7 & EPTA & 1.6 & $770 \times 630 \times 155$ & 440 & 20 & 103 & 506 & 83 & - & - \\
\hline \multirow[t]{2}{*}{8} & Os & 1.4 & $780 \times 745 \times 255$ & 431 & 56 & 171 & 307 & 52 & 78 & 6.4 \\
\hline & & & & $\begin{array}{l}468 \pm 65 \\
(\mathrm{SD})\end{array}$ & & & $\begin{array}{l}376 \pm 133 \\
(\mathrm{SD})\end{array}$ & $\begin{array}{l}55 \pm 16 \\
(\mathrm{SD})\end{array}$ & & $\begin{array}{l}5.2 \pm 0.67 \\
(\mathrm{SEM})\end{array}$ \\
\hline
\end{tabular}

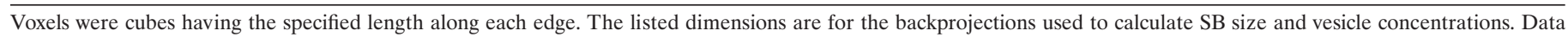
for the two SBs at synapse 4 are listed. At synapses 1 and 3, a second backprojection encompassing a larger volume was computed also (data not shown).

classified as docked if no space could be seen between the vesicle membrane and plasma membrane in at least one $x-y$ plane. The plane of contact was sometimes different from the plane of largest cross section (i.e., if the plasma membrane was curved or not perpendicular to the $x-y$ plane). Excluded from the docked category were vesicles connected to the plasma membrane by filaments but not touching it and vesicles that touched the thick portion of the presynaptic density without contacting the plasmalemma.

A sphere was fit to each SB by first fitting a circle to the profile of the SB traced in each $x-y$ plane and then calculating a weighted leastsquared-error fit of a sphere to four points on each circle. The data points were weighted by the radius of each circle, thereby placing less importance on data from sections that grazed the SB, which were difficult to trace accurately. We calculated the fraction of the SB captured in each reconstruction by measuring the area it delimited on the fitted sphere. The number of SB-associated vesicles was divided by the fraction of the $\mathrm{SB}$ reconstructed to estimate the total number of SB-associated vesicles.

We measured the concentration of vesicles in the cytoplasm by counting all vesicles in the three-dimensional reconstruction and then dividing by the reconstructed volume after subtracting the volumes of the postsynaptic cell, extracellular space, the SB, and mitochondria in each $x-y$ plane, using National Institutes of Health Image software. We also used conventional TEM micrographs acquired at a magnification of 22,000$28,000 \times$ to estimate vesicle concentrations. All vesicles in a section were counted, and the result was divided by the volume of cytoplasm in the section. The cytoplasmic volume was calculated, again with National Institutes of Health Image, by subtracting the nuclear and mitochondrial areas from the total area of the image and then multiplying by the effective section thickness. The effective section thickness was estimated from the interference color of the section, allowing for the fact that some vesicles with centers outside the section were included in the count. Assuming that vesicle outer diameters are $\sim 40 \mathrm{~nm}$ and that we counted all vesicle profiles having diameters larger than $\sim 20 \mathrm{~nm}$, we estimated the effective section to be $\sim 17 \mathrm{~nm}$ thicker on each face. Thus, a $50 \mathrm{~nm}$ section was considered to have an effective thickness of $\sim 85 \mathrm{~nm}$.

To estimate the total number of vesicles per cell, we assumed that hair cells are cylinders and measured their height and diameter in lowmagnification TEM images. The cytoplasmic volume was calculated by subtracting the nuclear volume from the total volume, assuming the nucleus to be either a cylinder or a cone (hair cell nuclei often appear triangular in thin sections; Roberts et al., 1990), depending on the shape of the nucleus in the micrograph. We assumed that hair cells have 20 afferent synapses each (Roberts et al., 1990), that the vesicle concentration within $1 \mu \mathrm{m}$ of each synapse was the average concentration measured in synaptic reconstructions, and that farther away the vesicle concentration was that measured in nonsynaptic regions of the cell. Because the hair cell volume estimates included mitochondrial volumes, we used vesicle concentrations that were not corrected for the space occupied by mitochondria for these calculations.

We expressed vesicle concentrations as a percentage of the maximum packing density, assuming vesicles are spheres having an outer diameter of $40 \mathrm{~nm}$. In two dimensions (on the surface of the SB or on the plasma membrane), dense-packed vesicles were assumed to be arranged hexagonally, each having the footprint of a hexagon just large enough to contain a $40 \mathrm{~nm}$ circle. To calculate the maximum number of vesicles that could fit around a SB, we divided the surface area at a radius equal to the radius of the fitted sphere plus the average tether length $(20 \mathrm{~nm})$ plus the vesicle radius $(20 \mathrm{~nm})$ by the hexagonal footprint of a single vesicle. In three dimensions (in cytoplasm), dense-packed vesicles were assumed to occur in a three-dimensional hexagonal array.

The spatial distribution of vesicles docked at the plasma membrane was calculated by projecting the centers of all docked vesicles onto a plane defined by three corners of the traced membrane and then rotating and translating the coordinate system so that the membrane lay in the $x-z$ plane, with the origin located at the projected center of the sphere fit to the SB. Arcs $1 \mathrm{~nm}$ wide were drawn about the origin, and the number of vesicle centers on each arc was divided by the area of membrane encountered. The radius was incremented in $1 \mathrm{~nm}$ steps from $1 \mathrm{~nm}$ to beyond the furthest edge of the membrane, and the data were pooled into 100 nm bins.

\section{RESULTS}

\section{Three-dimensional reconstruction of synapses}

We used electron tomography to reconstruct eight afferent synapses (Table 1) and one nonsynaptic region in frog saccular hair cells. A thick section containing the region of interest was imaged in the IVEM at a series of tilt angles, and the micrographs were digitized and backprojected to generate a three-dimensional rectangular solid composed of cubic voxels (see Table 1 for dimensions) that usually were viewed as a stack of $x-y$ planes, each one voxel thick (see Materials and Methods). Figure $1 A$ shows a subregion of one $x-y$ plane through a synapse. The computed sections resembled conventional transmission electron micrographs of this tissue (compare with Fig. $8 A$ ). The electron-dense $\mathrm{SB}$ is surrounded by a halo of small ( $\sim 35 \mathrm{~nm}$ diameter) clear-core vesicles and is situated in the hair cell cytoplasm at a distance of one to two vesicle diameters above the plasma membrane, with the synaptic cleft and postsynaptic plasma membrane beneath. The cytoplasm also contains numerous small clear-core vesicles not associated with the SB and occasional clathrin-coated vesicles (Fig. 1B).

Vesicles in the monolayer surrounding the SB were denoted "SB-associated" and all others as "outlying vesicles." Many of the SB-associated vesicles were tethered to it by one or more thin 

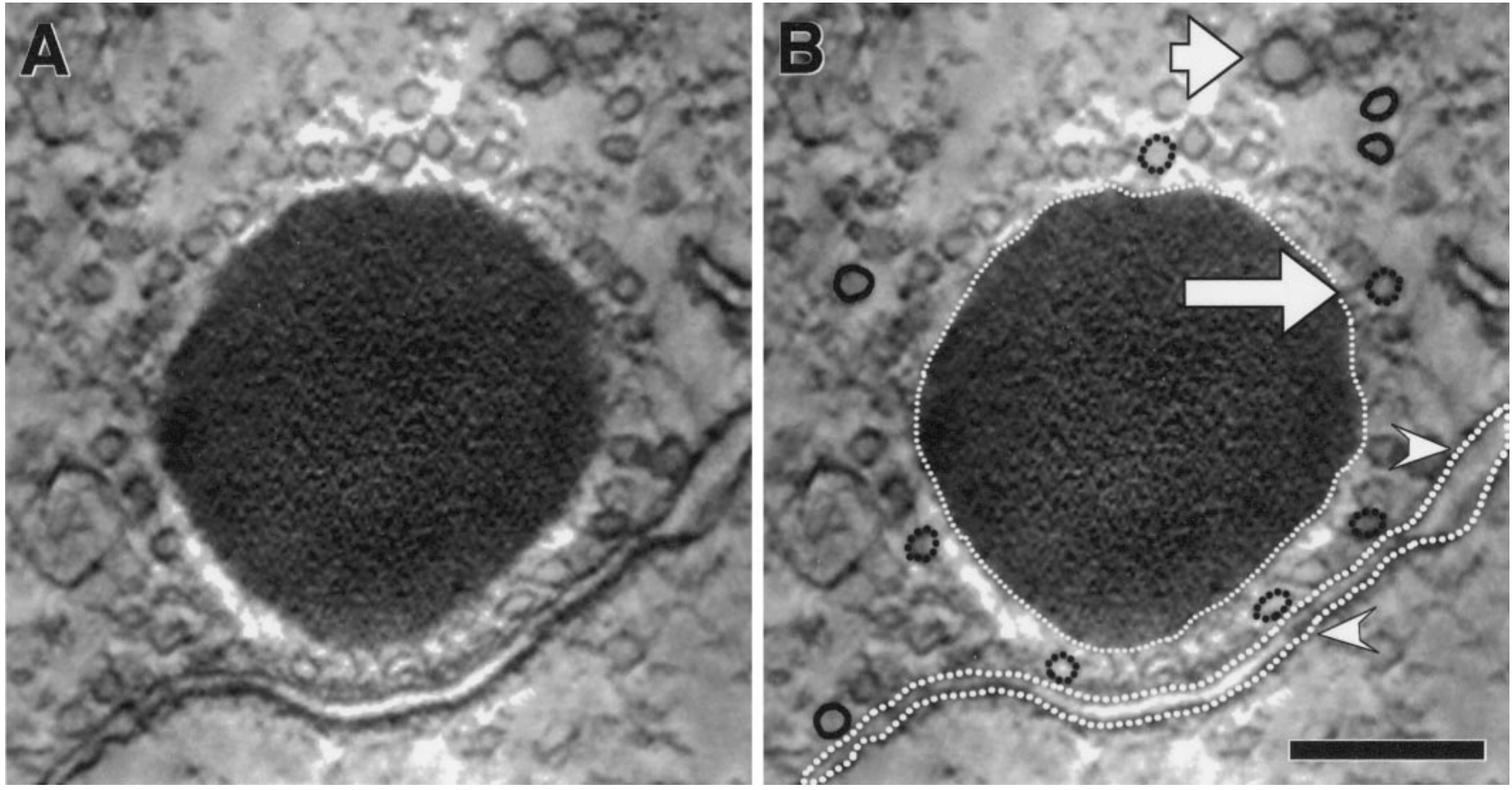

Figure 1. A slice through an osmium-stained hair cell afferent synapse, reconstructed by electron tomography. $A$, An $x-y$ plane through the reconstructed volume. $B$, Same plane as $A$, showing traced SB (thin dotted white line), SB-associated vesicles (dotted black lines), outlying vesicles (solid black lines), and the presynaptic (rightward arrowhead) and postsynaptic (leftward arrowhead) plasma membranes, which were rendered as a single object (thick dotted white line) joined at the edges of the plane. For clarity, tracing lines are shown thicker than they appeared in the tracing program. Also visible are coated vesicles (short arrow) and a tether attaching a vesicle to the SB (long arrow). The white areas immediately adjacent to osmium-stained SBs were probably artifacts of the backprojection computation related to the steep contrast gradient at the border of the darkly stained SB. The effect was not seen near less electron-dense SBs stained with EPTA; synapse 1. Scale bar, $200 \mathrm{~nm}$.

filaments (Figs. 1B, 10). Tethers on other SB-associated vesicles may have been present, but not resolved, particularly if they did not lie in an $x-y$ plane. The observation that a monolayer of vesicles was retained on the surface of the SB after cell lysis (see Fig. $10 E$ below) suggests that all SB-associated vesicles are bound to it in some way. Therefore, we included all vesicles within $30 \mathrm{~nm}$ of the SB surface as SB-associated. This distance is less than a vesicle diameter and is similar to the longest tethers that we observed $(28 \mathrm{~nm})$.

Figure $1 B$ illustrates the procedure used to trace vesicles and other structures for rendering in three dimensions. The SB and the plasma membranes were traced in each plane in which they appeared, whereas each vesicle was traced only in the plane in which it was largest. Thus only 10 vesicles (six SB-associated vesicles and four outlying vesicles) were traced in this plane of section. Four of the traced vesicles were categorized as "docked" (in contact with the plasma membrane; see Materials and Methods). Both SB-associated and outlying vesicles were included in the category of docked vesicles.

\section{Synaptic body topography and morphology}

Figure $2 A$ shows the reconstruction of the synapse in Figure 1, which displays features typical of all of the synapses analyzed. Vesicles were rendered as spheres and the traced organelles as surfaces to reveal their three-dimensional structure and spatial relationships. The SB had no visible internal substructure and was rendered as a hollow object to better show the distribution of vesicles around it and to reveal its shape, a concave hemisphere in this stereo pair. The SB was reconstructed incompletely because it was cut at the edge of the physical section. Vesicles were distributed across the entire reconstructed surface of the SB without any apparent pattern. At several synapses the plasma membrane followed the curve of the lower portion of the SB, creating a bulge in the surface of the cell that also has been seen by freeze-fracture in saccular hair cells from goldfish (Hama, 1980) and frog (Jacobs and Hudspeth, 1990). This bulge may expand during exocytosis (Hudspeth and Issa, 1996).

In previously published TEM images of frog saccular hair cells, SBs were always round in cross section (Jacobs and Hudspeth, 1990; Roberts et al., 1990; Issa and Hudspeth, 1994; Parsons et al., 1994), suggesting that they are spherical. Our reconstructions concurred with this interpretation. Figure $2 B$ shows a rendering of the plasma membrane, SB, and fitted sphere at another synapse. The SB is a lighter color where the semitransparent sphere overlaps it and darker where it overlaps the sphere. Figure 2, $C$ and $D$, shows that the $\mathrm{SB}$ was well fit by the sphere when it was viewed transected along two perpendicular planes. Each of nine reconstructed $\mathrm{SBs}$, including two that were more than two-thirds complete (Figs. $2 B-D, 7 B$ ), were well fit by spheres. From these fits the SB diameter was estimated to be $468 \pm 65 \mathrm{~nm}$ (mean \pm $\mathrm{SD}, n=9$; Table 1).

One reconstructed synapse contained two SBs (Table 1, synapse 4) that were so close together that some vesicles were associated with both. Only one of the two bodies was adjacent to the plasma membrane. It is possible that the second body was associated with an active zone outside the reconstructed volume, although this would be inconsistent with the simple cylindrical shape typical of these hair cells. Alternatively, the second body may be an example of the heterotopic SBs that have been re- 

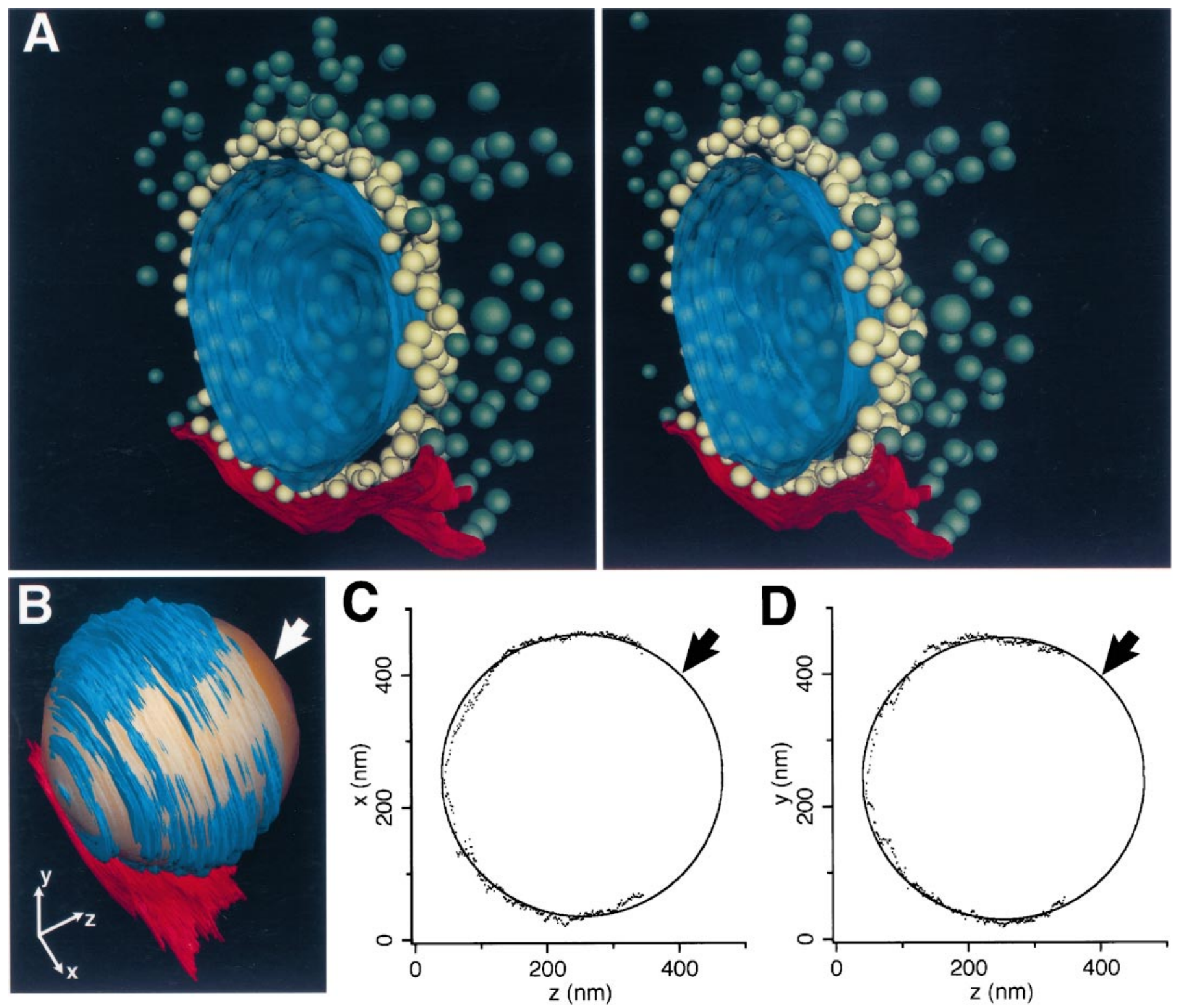

Figure 2. Three-dimensional structure of synaptic organelles. A, Stereo pair rendering of the partially reconstructed synapse shown in Figure 1. The SB (blue) is open on the face that met the edge of the section. SB-associated vesicles (yellow) coat the surface of the SB and are visible through its semitransparent wall. Outlying vesicles ( green) fill the adjacent presynaptic cytoplasm, which is delimited by the synaptic cleft (red), defined as the region in which pre- and postsynaptic membranes were in closest proximity. The slice through this synapse that is shown in Figure 1 was taken parallel to, and a short distance from, the open face of the SB. In black and white photocopies of these color figures the SB-associated vesicles appear lighter than the outlying vesicles. To view a stereo pair, hold the figure at forearm length and focus beyond the plane of the page; then merge the two panels until a central three-dimensional image appears. $B-D$, SBs were approximately spherical. $B$, Rendering of a semitransparent sphere (gold, arrow) fit to a partially reconstructed SB (blue). The region of the plasma membrane that formed the synaptic cleft is shown in red; vesicles were omitted for clarity. $C, D$, Shown is the fit of the sphere (smooth line, arrows) to the SB at each 1.3-nm-thick plane (dots) in the $x-z(C)$ and $y-z(D)$ planes passing through the center of the sphere. The dots are the edges of circles fit to the traced SB in each plane (see Materials and Methods); synapse 6.

ported to occur, complete with attached vesicles, in other hair cells (Miller and Beck, 1987). The second body at this synapse did not differ significantly from the average of the other eight in mean diameter, vesicle number, or vesicle packing density (Table 1).

\section{Vesicle concentrations}

To determine whether the number of SB-associated vesicles is sufficient to account for the largest evoked capacitance increases reported in these cells (Parsons et al., 1994), we counted all of the vesicles on the surface of each reconstructed SB (Table 1). After correcting for the missing portions of the spheres, we calculated an average of $376 \pm 133$ (mean $\pm \mathrm{SD}, n=9$ ) vesicles per whole SB (Table 1) and an average packing density of $55 \%$ of the carrying capacity of the SB. The packing density varied considerably among synapses $(26-83 \%$; Table 1$)$.

In a separate series of experiments we attempted to deplete the pool of SB-associated vesicles by depolarizing hair cells for up to $30 \mathrm{~min}$ in 22,45 , or $80 \mathrm{~mm} \mathrm{~K} \mathrm{~K}^{+}$before fixation. Even if frog saccular hair cells have a very negative $E_{\mathrm{K}}$ of $-90 \mathrm{mV}$ in normal $\left(2 \mathrm{mM} \mathrm{K}^{+}\right.$) extracellular saline, these $\mathrm{K}^{+}$concentrations are expected to cause sufficient depolarization (to $-30,-12$, and +2 

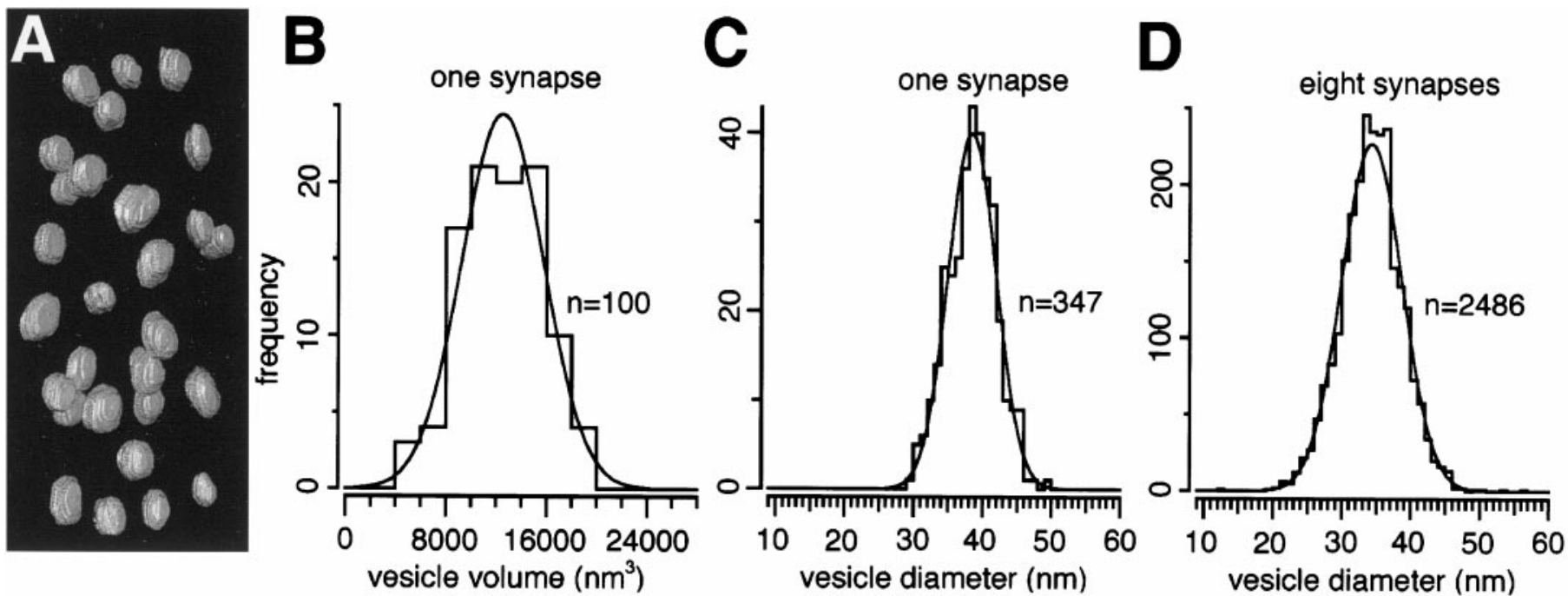

Figure 3. Distribution of vesicle diameters and volumes. $A$, Renderings of some of the vesicles described in $B$. $B-D$, Distributions of vesicle volumes $(B)$ and diameters $(C, D)$ fit by Gaussians (smooth lines). The smallest ticks on the abscissa denote the bin size. $B$, Vesicle volumes at a single synapse (synapse 6). $C$, All vesicle diameters at a single synapse (synapse 5). $D$, All vesicle diameters from all eight synapses. The vesicle total numbers six fewer here than in Table 1 because these were shared by two SBs at synapse 4 . Here they were counted only once.

$\mathrm{mV}$, respectively) to activate massively the presynaptic $\mathrm{Ca}$ current; in the intact epithelium this current is $\sim 90 \%$ activated at $-30 \mathrm{mV}$, reaches peak at $-20 \mathrm{mV}$, and does not inactivate (Armstrong and Roberts, 1998). The presence of coated pits and coated vesicles indicated that membrane recycling was under way, but under all conditions SBs retained their monolayer of vesicles (see Fig. $8 A$ ).

To investigate the possibility that mechanisms other than binding to the SB may serve to concentrate vesicles at synapses, we determined the concentration of vesicles in the cytoplasm near and far from synapses. Near synapses, we counted all outlying vesicles in the reconstructed synaptic volumes. We estimate the average concentration of outlying vesicles to be $5.2 \%$ of densepacked in the $0.97 \mu \mathrm{m}^{3}$ of available cytoplasm (see Materials and Methods) sampled in the vicinity of synapses (Table 1). These vesicles were at most $700 \mathrm{~nm}$ from the SB, the distance to the farthest corner in the largest reconstructed volume (synapse 4). We used two methods to determine the concentration of vesicles farther from synapses. First, we surveyed $50 \mathrm{~nm}$ sections of two hair cells by using TEM, and we collected 22 micrographs. These sections contained a total of 4038 small clear-core vesicles in 14 $\mu \mathrm{m}^{3}$ of cytoplasm (see Materials and Methods) from which we estimate their concentration to be $1.2 \pm 0.07 \%$ (mean \pm SEM) of dense-packed. Second, we used electron tomography to reconstruct a nonsynaptic region of hair cell cytoplasm adjacent to the plasma membrane at an unknown distance from any synapse (see Fig. $4 F$ ) but in the basal portion of the cell in which synapses are common (Roberts et al., 1990). In this reconstruction we counted 91 vesicles in $0.96 \mu \mathrm{m}^{3}$ of cytoplasm, which corresponds to a concentration of $0.4 \%$ of dense-packed. Although we have only a single reconstruction of a nonsynaptic region, this lowmagnification tomogram encompassed nearly the same volume as all of the synaptic volumes combined. The $\sim$ threefold difference between the two estimates of extrasynaptic vesicle densities remains unexplained. It might reflect statistical variation caused by small sample size, error in determining the section thickness in TEM, or differences between the two methods used to count vesicles. In any event, both measurements show that the vesicle concentration was higher in the neighborhood of the synapse than elsewhere in the cell (four- to 13-fold; compare outlying vesicle densities in Figs. $2 A$ or $7 B$ with $4 F$ ).

Vesicles near synapses comprised a small fraction of the total number per cell. Using the two estimates of cytoplasmic vesicle densities given above and the average volume of three hair cells (2.2 pl, excluding the nucleus), we estimate that a hair cell contains 200,000-600,000 small clear-core vesicles.

\section{Vesicle sizes}

We measured the mean diameter and volume of synaptic vesicles as well as the variance of the distributions around the mean. These measurements are important for interpreting the measured rates of capacitance increase in terms of vesicle fusions per second and for testing hypotheses concerning the source of quantal variation (see Discussion).

In the reconstruction with the finest voxel size $(1.3 \mathrm{~nm}$; synapse $6)$, we computed the volumes of 100 vesicles by tracing the perimeter of each vesicle in all of its planes. Figure $3 A$ shows renderings of some of these vesicles. Volumes were distributed with a $C V$ of $26.1 \%$ about a mean of $12,500 \mathrm{~nm}^{3}$ (Fig. $3 B$ ). We examined two sources of artifact that could have increased the width of this distribution. The sampling error caused by the finite voxel size was found to be negligible; vesicle profile diameters had a $C V$ of $0.13 \%$ when we simulated cutting 10,000 identical $40 \mathrm{~nm}$ vesicles into randomly spaced $3 \mathrm{~nm}$ slices, the thickest used in this study. A second, more substantial variance was attributable to tracing errors. We determined the magnitude of this error by retracing two of the vesicles 25 times each and found a $6.0 \% \mathrm{CV}$ attributable to tracing error. Correcting for tracing error (see Materials and Methods) reduced the volume $C V$ to $25.7 \%$. This value agreed well with the $C V$ of the cubed diameters of the same 100 vesicles $(C V=24.5 \%)$. We therefore used the much more economical measurement of vesicle diameters to estimate the $C V$ of vesicle volumes at each of the reconstructed synapses. We also found that the reconstructed vesicle volumes were $\sim 34 \%$ smaller than the volumes of the corresponding spheres. This difference is 
a reflection of the flattened shapes of the vesicles, perhaps because of shrinkage during fixation.

Figure $3 C$ shows the distribution of vesicle diameters and Gaussian fit $(38.5 \pm 3.5 \mathrm{~nm}$, mean $\pm \mathrm{SD})$ for all vesicles $(n=347)$ at a single synapse. The diameters of SB-associated vesicles $(38.8 \pm 3.4 \mathrm{~nm} ; n=139)$ and outlying vesicles $(38.3 \pm 3.5 \mathrm{~nm} ; n=$ 208) at this synapse were not significantly different, as was true at five of seven synapses. Pooling all of the vesicles from all eight synapses gave a mean diameter of $34.3 \mathrm{~nm}$. The $C V$ of vesicle diameters was $9.0 \%$ at the synapse shown in Figure $3 C$, and the average $C V$ of the eight synapses was $10.2 \%$ (range, $8.8-12.8 \%$ ). We averaged the $C V S$ from individual synapses rather than pooling data across synapses because variation between tissue samples, perhaps because of differences in fixation or handling, substantially increased the variance around the group mean $(\mathrm{CV}=$ 12.7\%; Fig. $3 D$ ). As with the volume distributions, the component of the variance contributed by the tracing method was measured by retracing 46 vesicle profiles $15-96$ times each. An average $C V$ of $5.1 \%$ was attributable to the method. The corrected $C V$ of the vesicle diameter distribution for the synapse shown in Figure $3 C$ was $7.4 \%$. The average corrected $C V$ of vesicle diameters for all eight synapses was $8.8 \pm 0.6 \%$ (mean \pm SEM, $n=8$ ), which corresponds to a $C V$ of vesicle volumes of $26 \pm 2 \%$ (mean \pm SEM, $n=8)$.

\section{Docked vesicles}

At each reconstructed synapse we mapped the three-dimensional distribution of morphologically docked vesicles in relation to the $\mathrm{SB}$, the presynaptic density, and each other (Fig. $4 A, C, E$ ). The identification of morphologically docked vesicles was aided by the ability to scan through the $z$-series looking for a point of contact between the vesicle and the membrane (see Materials and Methods); we estimate that vesicles classified as "docked" were $<10 \mathrm{~nm}$ from the membrane; resolution was superior to this in most reconstructions. Many docked vesicles were present beneath each SB (Fig. 4B), as has been proposed for the ribbon synapses in bipolar cells (Mennerick and Matthews, 1996; von Gersdorff et al., 1996) and rod photoreceptors (Rao-Mirotznik et al., 1995). Most $(88 \%)$ of these vesicles also were classified as SB-associated (Fig. 5). In three synapses that we analyzed in detail (see Fig. 4), we counted between 17 and 31 (78 total) SB-associated docked vesicles, some of which clearly were tethered to the SB (see Fig. 10C).

The presynaptic densities observed in hair cells by TEM may align with particle arrays seen in freeze-fracture replicas of the presynaptic membrane (Hama, 1980; Jacobs and Hudspeth, 1990). These particles have been observed to occur in rows a vesicle's breadth apart (Hama, 1980; Jacobs and Hudspeth, 1990; Roberts et al., 1990). In Rana pipiens saccular hair cells it has been proposed that many of these particles correspond to the voltage-gated $\mathrm{Ca}$ channels that regulate synaptic exocytosis (Roberts et al., 1990). In our survey of several hundred TEM micrographs of hair cell synapses (D. Lenzi and W. Roberts, unpublished observations), the presynaptic density was seen either as a band (see Fig. 8A) or in patches (see Fig. 10D,E), consistent with stripes cut along their length or across their width. Figure 4 shows a similar pattern at the three partially reconstructed active zones. Although docked vesicles were not organized in neat rows between the presynaptic densities as proposed for other hair cells (Hama, 1980), 54\% of the 78 docked SBassociated vesicles were docked within $50 \mathrm{~nm}$ of a presynaptic density, and one-half of these touched a presynaptic density. Some of the remaining vesicles on the edge of the reconstruction probably were docked within $50 \mathrm{~nm}$ of areas of presynaptic density outside the volume, so $54 \%$ is a lower limit. In a spatial model of the $\mathrm{Ca}^{2+}$ concentration at the hair cell active zone (Roberts, 1994), vesicles docked within $50 \mathrm{~nm}$ of an open Ca channel are predicted to experience $>20 \mu \mathrm{M} \mathrm{Ca}{ }^{2+}$ during a depolarization, a concentration sufficient to trigger exocytosis at ribbon synapses in retinal bipolar cells (Heidelberger et al., 1994; von Gersdorff and Matthews, 1994) (for a substantially lower estimate, see Lagnado et al., 1996) and photoreceptors (Rieke and Schwartz, 1996). During a large depolarization that opens many Ca channels, all vesicles docked beneath the SB would experience $\mathrm{Ca}^{2+}$ concentrations sufficient to trigger exocytosis (Roberts, 1994).

We also observed outlying vesicles docked at the plasma membrane. Some of these were near the SB (Fig. $4 A-E$ ), but only two of 74 docked outlying vesicles were within $50 \mathrm{~nm}$ of the presynaptic density. Additional docked vesicles also were seen much farther away from the active zone. Figure $4 D$ shows a second reconstruction of the same synapse shown in Figure $4 C$ but extending $\sim 1200 \mathrm{~nm}$ away from one side of the active zone; Figure $4 F$ shows a reconstruction of a nonsynaptic region of membrane. In each, docked vesicles were observed far from the active zone and therefore far from the cluster of presynaptic $\mathrm{Ca}$ channels (Roberts et al., 1990; Issa and Hudspeth, 1994; Martinez-Dunst et al., 1997).

To test the hypothesis that docking occurs preferentially at synapses, we mapped the density of docked vesicles as a function of distance from the active zone. Figure 5 shows pooled data from three active zones. The white bars show the density of docked SB-associated vesicles, which peaked at $40 \%$ of dense-packed, and then fell to zero beyond the radius of the SB. The density of docked outlying vesicles (Fig. 5, dark bars) declined just beyond the edge of the SB to near zero far away. Some outlying vesicles were seen as close as $100 \mathrm{~nm}$ from the origin, where the SB lay at least two vesicle diameters above the plasma membrane. A few vesicles were seen beyond $900 \mathrm{~nm}$ from the origin, but these bars are contributed by only eight vesicles, seen at the left edge of the membrane in Figure $4 D$, and the rise in the curve may be attributable to the small sample of membrane this far from the active zone, mapped from only a single reconstruction.

To infer a binding association between morphologically docked vesicles and the plasma membrane, it is necessary to show that the concentration of vesicles at the membrane is greater than in the neighboring cytoplasm. In the nonsynaptic reconstruction (see Fig. $4 F$ ) the density of docked vesicles was $1.7 \%$ of dense-packed, which far exceeded the expected value $(0.13 \%$, even if all vesicles within $10 \mathrm{~nm}$ of the membrane were counted as docked), based on the vesicle concentration in nonsynaptic regions of the cytoplasm. A similar analysis at reconstructed synapses showed that here also the average density of docked outlying vesicles (10\% of densepacked) is far above the value of $1.7 \%$ predicted from the cytoplasmic concentration. Our data therefore indicate a specific binding interaction between vesicles and the plasma membrane both near and far from the synapse. The presence of docked vesicles far from the SB indicates that contact with the SB is not required for the morphological docking of vesicles.

We calculated the average number of vesicles docked at an active zone by integrating the distribution in Figure 5 between the origin and the average radius of the active zone. Using the average particle array radius seen in freeze-fracture replicas at these same synapses (138 nm; Roberts et al., 1990) plus one vesicle diameter $(40 \mathrm{~nm}$, to allow for vesicles docked on the 

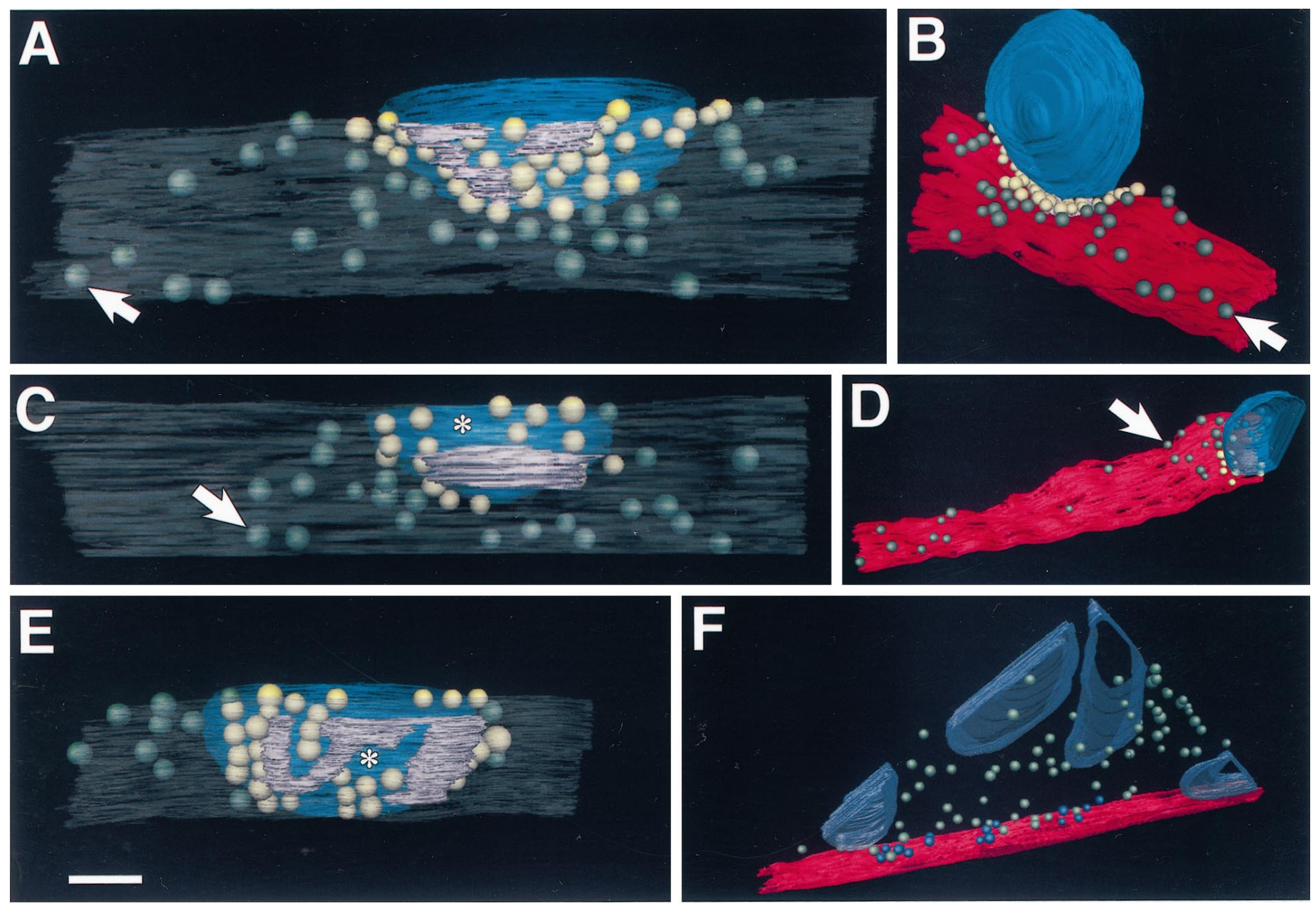

\section{Figure 4}
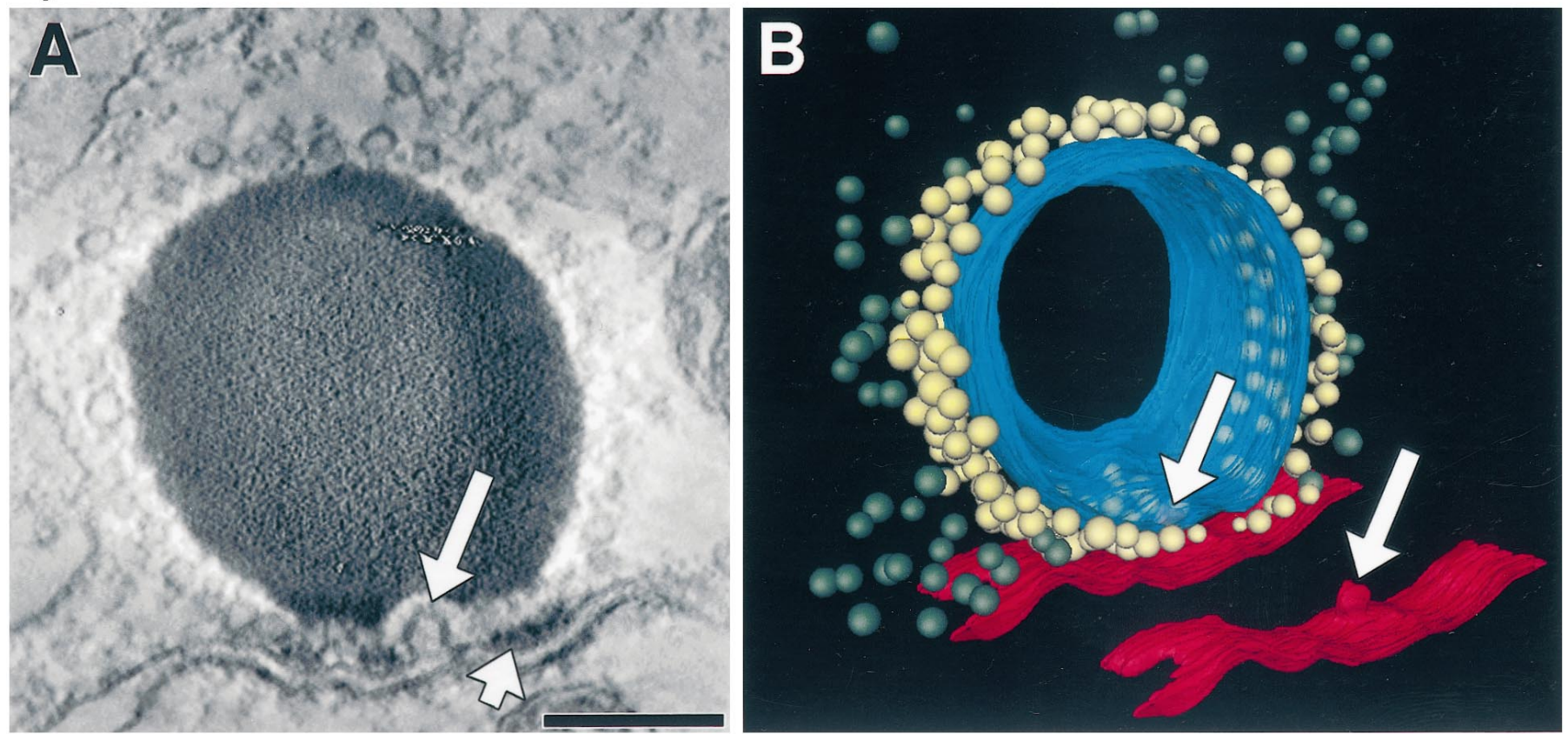

\section{Figure 6}

Figure 4. Spatial distribution of docked vesicles. SB-associated (yellow) and outlying (green) vesicles are shown at three synapses $(A, B$, synapse $1 ; C$, $D$, synapse $3 ; E$, synapse 2) in relation to the plasma membrane (gray), the SB (blue), and the presynaptic density (pink). Arrow pairs in $A / B$ and $C / D$ denote the same vesicle in both views of the same synapse. $A, C, E$, View through the mostly transparent plasma membrane of vesicles docked at it. Asterisks in $C$ and $E$ show the locations of omega-shaped invaginations in the presynaptic membrane. Scale bar (applies to $A, C, E$ ), $100 \mathrm{~nm}$. $B$, View of docked vesicles and the closed pole of the SB. D, Another tomogram of the synapse shown in $C$, revealing docked vesicles (Figure legend continues.) 


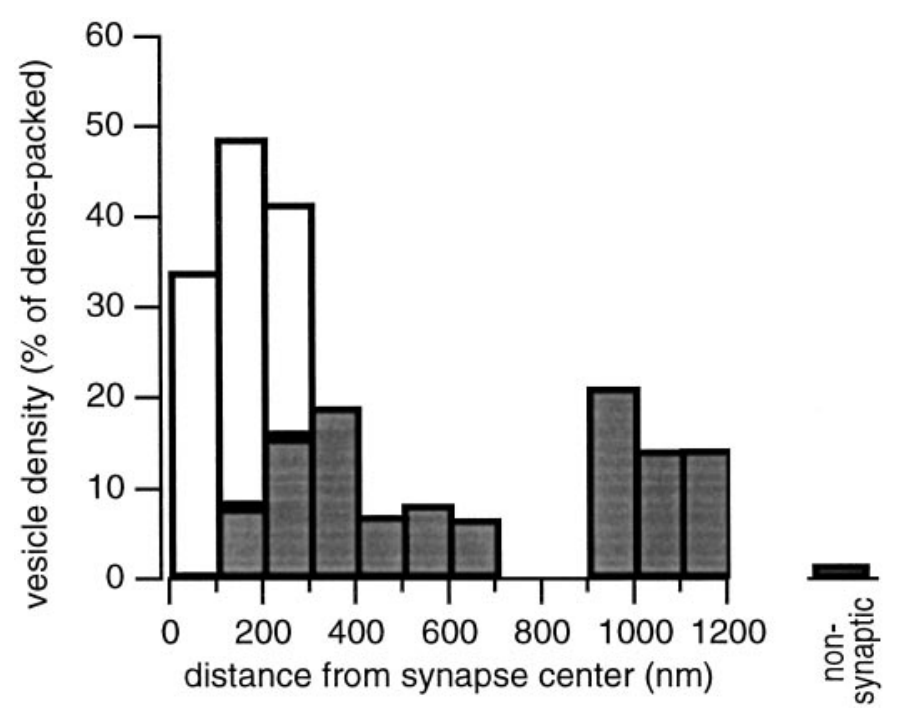

Figure 5. Density distribution of docked vesicles. Vesicle concentration on the plasma membrane, shown as a percentage of the maximum packing density (see Materials and Methods), is plotted as a function of the radial distance from the projected center of the SB. The envelope of the bar graph shows the distribution of the 152 docked vesicles from the three synapses shown in Figure 4. White bars show docked SB-associated vesicles $(n=78)$; dark bars show docked outlying vesicles $(n=74)$. The rightmost bar shows the docked vesicle density computed from a tomogram that did not include a synapse $(n=15$ vesicles $)$.

outside edge of the array) as the active zone radius, we estimate an average of 32 docked vesicles per active zone and a packing density of $42 \%$.

\section{Vesicles continuous with the plasma membrane}

Because electron tomography captures a large portion of the synapse in each reconstruction, it can reveal rare events that seldom are observed in single thin sections. We were interested particularly in omega profiles (putative sites of vesicle fusion or fission with the plasmalemma) that may help to clarify the role of the SB in the synaptic vesicle cycle and provide evidence of local membrane recycling. Although we did not stimulate transmitter release in these cells, we observed approximately one site per synapse (eight sites total) at which vesicle membranes were continuous with the plasma membrane. These fusion/fission sites may reflect a low level of continuous exocytic activity at hair cell synapses (for review, see Roberts et al., 1991; Lenzi and Roberts, 1994) or possibly glutaraldehyde-induced exocytosis (Smith and Reese, 1980).

Two of the eight omega profiles that we identified in the reconstructed synapses were situated directly beneath the SB in the spaces between the areas of presynaptic density (see asterisks in Fig. $4 C, E$ ). Figure $6 A$ is a tomographic section through the equator of the structure marked by the asterisk in Figure $4 E$, revealing an invagination of the plasma membrane situated between two areas of presynaptic density. The invagination is larger than neighboring clear-core vesicles and has several tethers linking it to a concavity on the underside of the SB. Strikingly similar features have been seen in conventional TEM images of frog saccular hair cells (Jacobs and Hudspeth, 1990), although their three-dimensional shapes could not be determined from single sections. On the basis of our three-dimensional reconstruction (Fig. 6B), we conclude that this class of structures may represent vesicular fusion or fission events. Figure $6 B$ also reveals that the concavity in the SB seen in Figure $6 A$ is a long furrow cut in cross section. The other omega profile that we found beneath a SB (see asterisk in Fig. $4 C$ ) had a more hemispherical profile, was the approximate size of SB-associated vesicles, and appeared to be tethered to the SB.

The other six omega profiles found in the reconstructed synapses were located near, but not beneath, SBs. These structures were seen in one osmium- and three EPTA-stained reconstructions. Figure $7 A$ shows a section through one such structure. Its three-dimensional shape appears in Figure $7 B$, along with a second profile not visible in $A$. Another example is visible in the stereo pair in Figure 9D (short arrow). Two other invagination sites had similar locations, lying near the SB, but not touching it. The perisynaptic location of all of these sites matches that of the dimples seen by freeze-fracture on the periphery of the active zone particle array in hair cells of Rana pipiens (Roberts et al., 1990) and other species (Hama, 1980; Jacobs and Hudspeth, 1990). The six perisynaptic omega profiles in our reconstructions had a variety of neck shapes, including a thin stalk (Fig. 7A), a narrow but short constriction (see Fig. $9 D$ ), or a tube almost as wide as the head of the invagination.

Because most of the perisynaptic invaginations seen in our tomographic reconstructions were in EPTA-stained tissue in which clathrin coats were not visible, we could not determine directly whether the reconstructed structures corresponded to coated pits. However, the observed range of sizes, locations, and shapes of these structures matched the coated pits that have been reported in previous TEM studies of hair cells (Gleisner et al., 1973; Hama and Saito, 1977; Leake and Snyder, 1987; Nadol, 1990) and those we observed in conventional TEM sections of cells that had been stimulated by $22 \mathrm{~mm} \mathrm{~K}^{+}$(Fig. 8A). The locations of coated pits and coated vesicles in $\mathrm{K}^{+}$-depolarized cells (Fig. $8 A$ ) suggest that endocytosis takes place (Mellman, 1996) next to the SB, outside the region of presynaptic density. To investigate whether endocytosis was also in progress in the unstimulated cells used for tomographic reconstructions, we labeled the endocytic pathway with an extracellular marker (microperoxidase). Figure $8 B$ shows that the lumens of two coated vesicles had been continuous with the extracellular space at some time during the $15 \mathrm{~min}$ exposure to microperoxidase in saline containing normal extracellular $\mathrm{K}^{+}$and $\mathrm{Ca}^{2}$ (see Materials and Meth-

$\leftarrow$

far from the SB, which is truncated by two edges of the volume. $F$, Nonsynaptic region of a hair cell showing vesicles in the hair cell cytoplasm ( pale green) and those docked (dark blue) at the plasma membrane (red). Mitochondrial outer membranes ( purple) are flat where truncated by the edge of the volume and reveal the wedge shape of this reconstruction.

Figure 6. Omega profile beneath the SB. $A$, An $x-y$ plane through an osmium-stained reconstructed synapse showing an omega-shaped invagination in the presynaptic plasma membrane that lies beneath the SB (long arrow) and adjacent to an area of presynaptic density (short arrow). White specks in the top portion of the SB are saturated pixels and are artifact. Scale bar, $200 \mathrm{~nm}$. B. Rendering of the same synapse showing the relationship of the omega profile (long arrows) to the partially reconstructed SB (blue), SB-associated vesicles (yellow), outlying vesicles (green), and the region of the plasma membrane that formed the synaptic cleft $(r e d)$. The membrane is a subregion of that shown in Figure $4 E$. The SB is open on two sides in this reconstruction because it spanned the thickness of the section. The membrane and omega profile also are shown alone; synapse 2. 


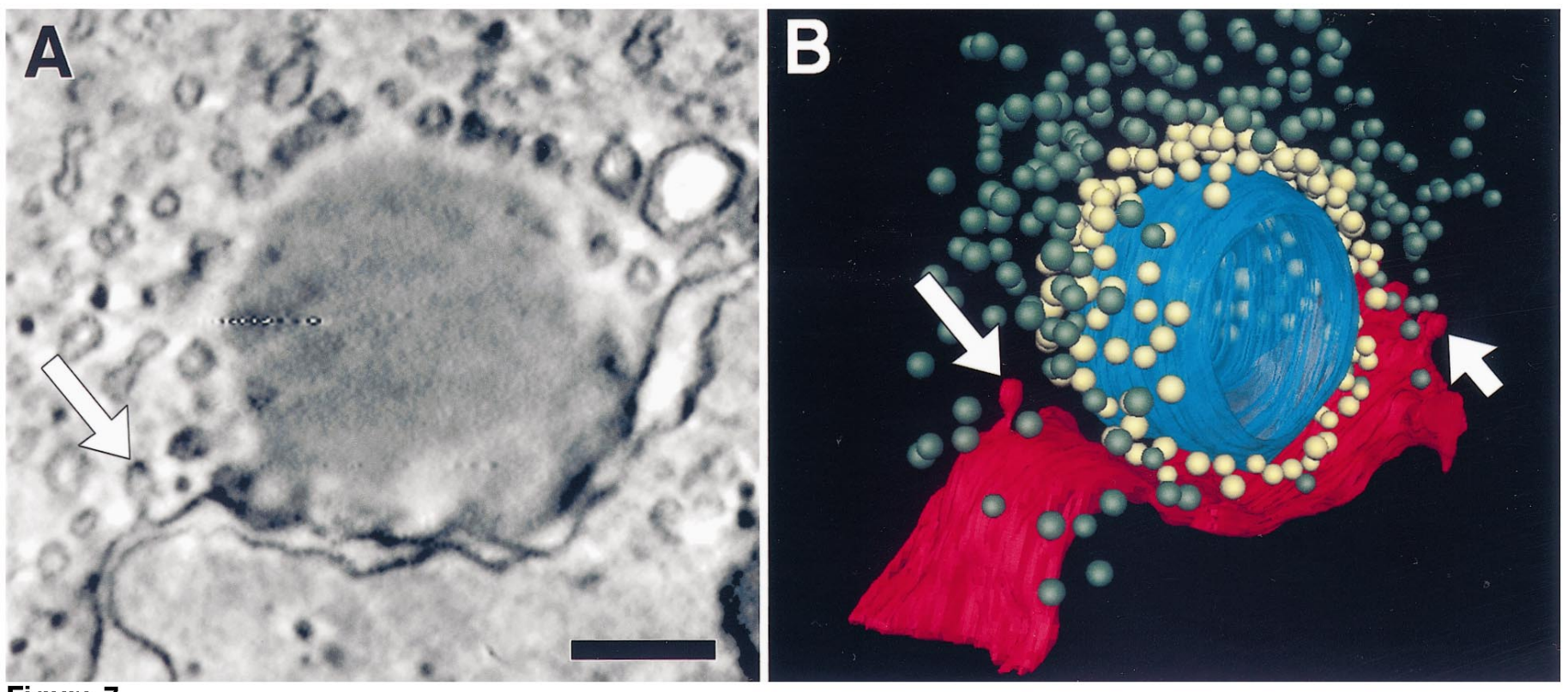

Figure 7
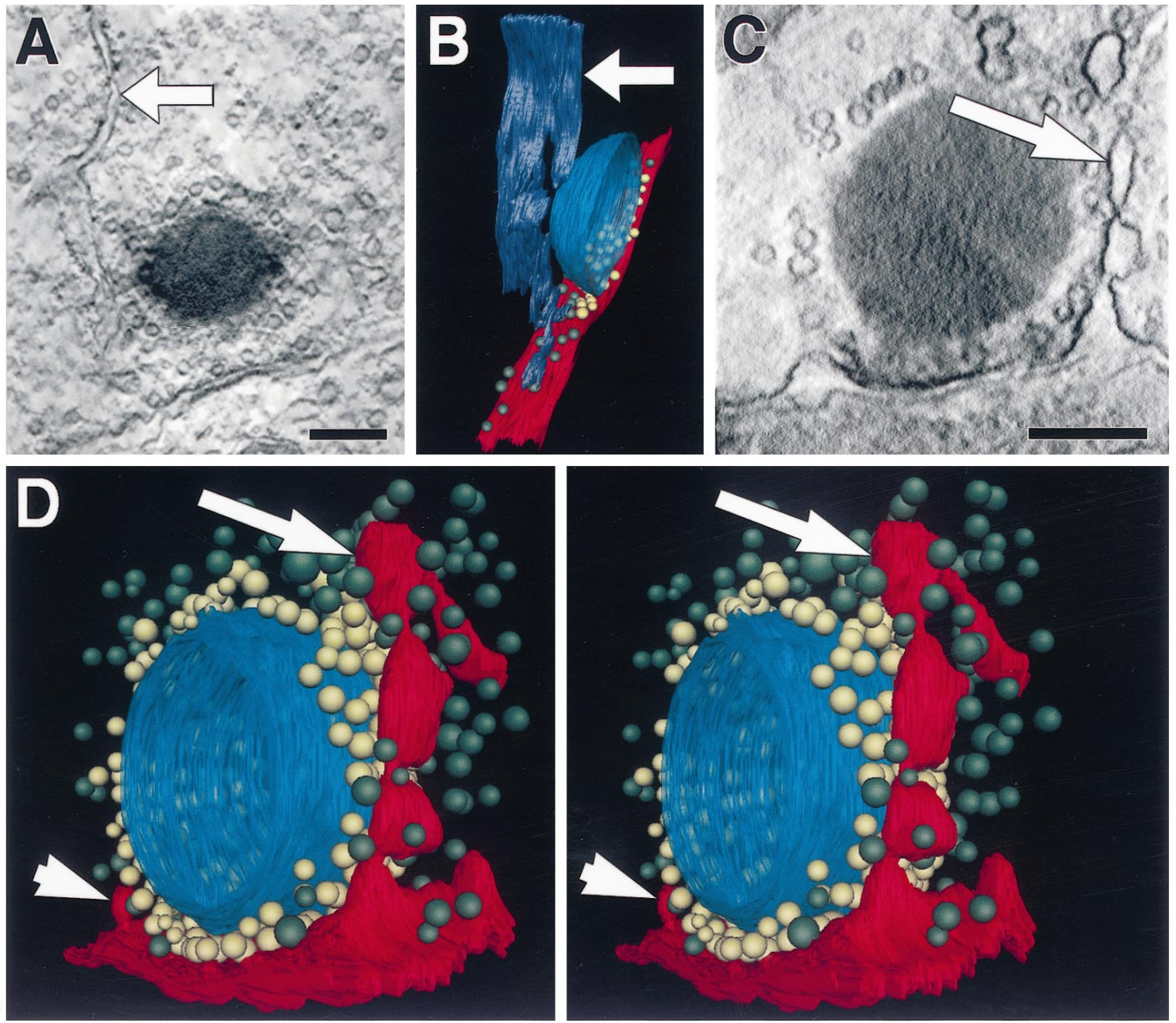

Figure 9

Figure 7. Elongated omega profiles near the SB. $A$, An $x-y$ plane through an EPTA-stained reconstructed synapse showing a vesicle formed from a stalk of the plasma membrane (long arrow) $165 \mathrm{~nm}$ from the SB. Scale bar, $200 \mathrm{~nm}$. B, Rendering showing this invagination (long arrow) in relation to the SB (blue), SB-associated vesicles ( yellow), outlying vesicles ( green), and the plasma membrane (red). Another similar invagination occurred on the other side of the SB (short arrow); synapse 5. 

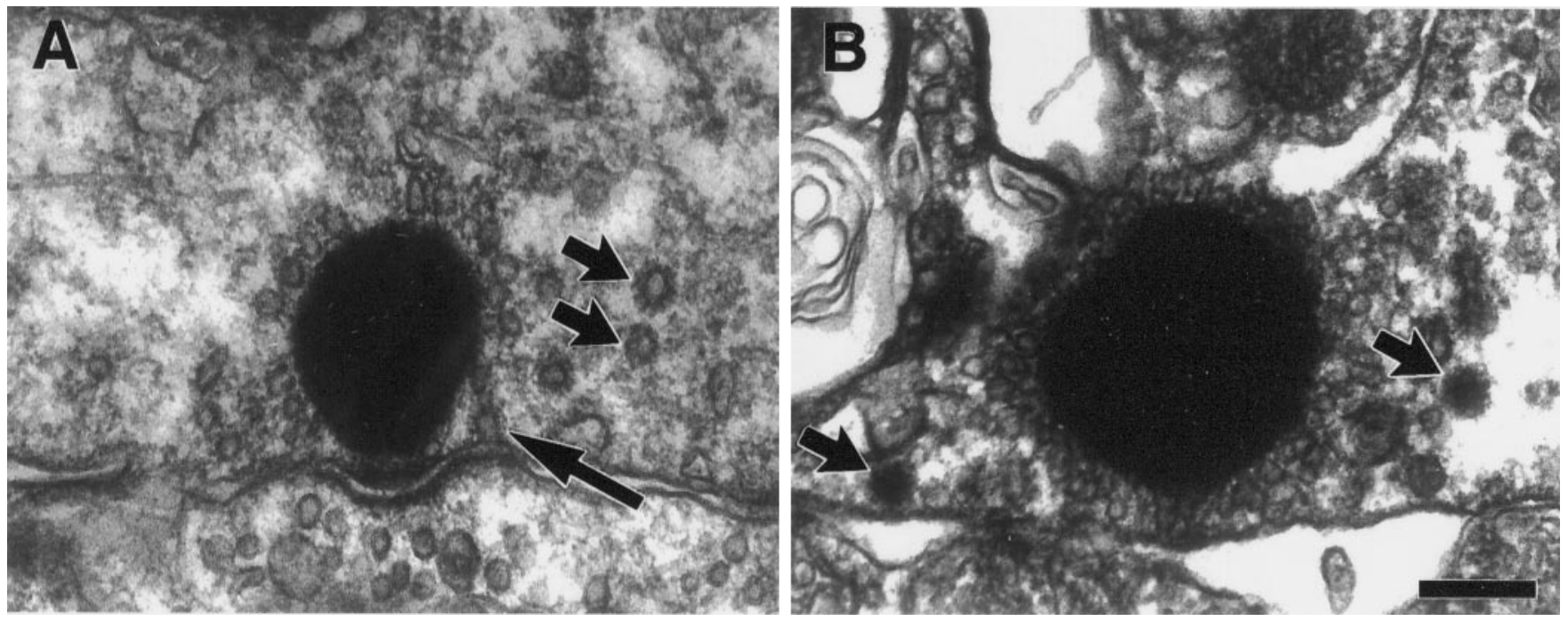

Figure 8. Endocytosis machinery near the SB. A, Conventional transmission electron micrograph of a hair cell afferent synapse depolarized in 22 mM $\mathrm{K}^{+}$saline. Numerous coated vesicles occur in the cytoplasm surrounding the SB (short arrows), and a coated pit in the plasma membrane is visible adjacent to the SB (long arrow). B, Conventional transmission electron micrograph of a synapse exposed to extracellular microperoxidase. Two coated vesicles contain the label (arrows). Scale bar, $200 \mathrm{~nm}$.

ods). This demonstrates that ongoing clathrin-mediated endocytosis can occur without stimulation under the same conditions as those used to prepare tissue for electron tomography.

\section{Tubules near the synaptic body}

Numerous small membranous compartments were present at all eight synapses, and larger membrane-bounded compartments were present at two of the reconstructed synapses (Fig. 9). At one synapse (Fig. 9C) we observed a deep infolding of the plasma membrane forming a lumpy tube, $40-65 \mathrm{~nm}$ across, that penetrated $600 \mathrm{~nm}$ into the cell. Rendering the structure (stereo image in Fig. 9D) showed it to be composed of connected lobes running alongside the SB amid the SB-associated and outlying vesicles. We did not observe any vesicles budding from its surface, but several lay adjacent to its membrane. At another synapse (Fig. $9 A$ ) a tubular profile resembling smooth endoplasmic reticulum extended from the top edge of the reconstruction to within $50 \mathrm{~nm}$ of the plasma membrane, but it was not continuous with the cell surface (Fig. 9B).

\section{Attachment of vesicles to the synaptic body and the synaptic body to the plasma membrane}

We measured the tethers with the highest contrast linking vesicles to the SB (see Figs. 1, 10A-C) and found an average length of $21 \pm 5.4 \mathrm{~nm}(\mathrm{SD}, n=10)$. Additional evidence that tethers do not exceed the vesicle diameter comes from the observation that only a monolayer of vesicles remained on the SB after cell lysis (see Fig. $10 E$ below). In photoreceptors the tethers are $20-50 \mathrm{~nm}$ in length (Rao-Mirotznik et al., 1995) and number three to five per vesicle (Usukura and Yamada, 1987). In bipolar terminals they are $25 \mathrm{~nm}$ long (von Gersdorff et al., 1996). Although most vesicles in our reconstructions had only one well stained tether, many had a second, less electron-dense filament linking it to the SB (all three vesicles with arrows in Fig. $10 A$; the left-arrowed vesicle in Fig. $10 \mathrm{~B}$ ), including vesicles docked at the active zone (Fig. 10C). Filaments also were seen between neighboring SBassociated vesicles (Fig. 10 $\mathrm{A}$ ), between docked and cytoplasmic vesicles, and between vesicles and the plasma membrane outside the active zone.

Figure $10 E$ shows that vesicles remain tightly bound to the SB when they are exposed to the high $\mathrm{Ca}^{2+}(4 \mathrm{~mm})$ in the extracellular saline. While furrowing the epithelium to break the diffusion barrier (see Materials and Methods), a few cells were broken open and most of their contents was lost (compare the cytoplasm of the hair cell with that of the undamaged postsynaptic cell), yet the SB retained its monolayer of vesicles. SBs appeared to be anchored to the presynaptic density by filaments (Fig. 10D), similar to those observed in other hair cells (Gleisner et al., 1973). Because SBs remained at the active zone in lysed cells, these filaments, like the vesicle tethers, were evidently stable in $4 \mathrm{~mm}$ $\mathrm{Ca}^{2+}$.

\section{DISCUSSION}

We found electron tomography to be a convenient method for reconstructing the presynaptic architecture in frog saccular hair cells. The good $z$-axis resolution compared with the thickness of TEM sections was important for visualizing vesicles beneath the SB and for identifying docked vesicles, particularly where the plasma membrane was not perpendicular to the plane of section. Three-dimensional reconstruction allowed us to identify extensive membrane-bounded structures at some synapses, count SBassociated vesicles and docked vesicles at each synapse, determine

$\leftarrow$

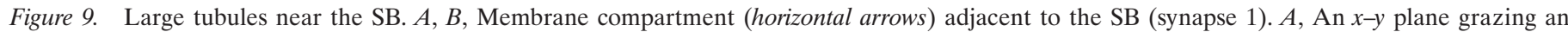

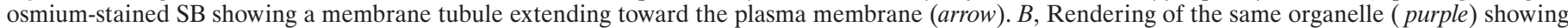

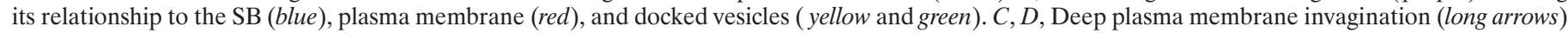

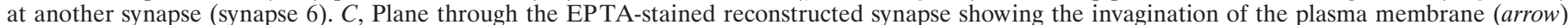

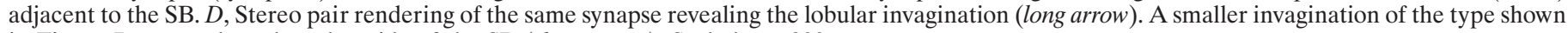
in Figure 7 occurred on the other side of the SB (short arrow). Scale bars, $200 \mathrm{~nm}$. 

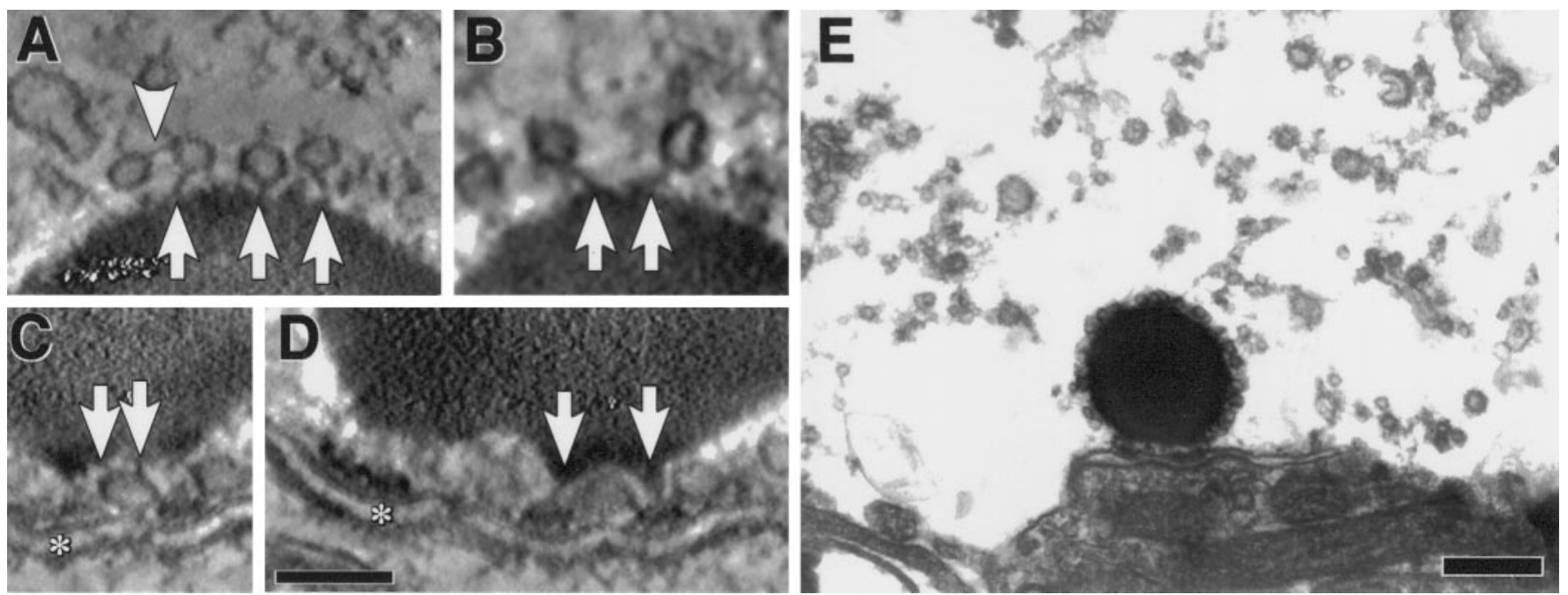

Figure 10. Tethers link the SB to vesicles and to the presynaptic density. $A-D$, Subregions of $x-y$ planes through osmium-stained reconstructed synapses. Each panel contains a portion of a SB. Scale bar, $100 \mathrm{~nm} . A, B$, Tethers linking vesicles to the surface of the SB (arrows) and to other vesicles (arrowhead in $A$ ). $C$, Two tethers (arrows) linking one docked vesicle to the SB. The vesicle is in contact with the presynaptic plasma membrane, which forms the synaptic cleft (asterisks in $C, D$ ). $D$, Two filaments (arrows) join the SB to the presynaptic density on the plasma membrane. $A, C, D$, Synapse 2 . $B$, Synapse 3. E, TEM micrograph of a synapse in a mechanically lysed hair cell. Although the presynaptic cytoplasm has lost most of its contents, the SB retains its monolayer of vesicles and remains at the active zone. Scale bar, $300 \mathrm{~nm}$

the variance in vesicle volume, and observe rare events such as putative endocytic sites and docked outlying vesicles.

It has been proposed that exocytic vesicles pass through a docking stage in which proteins comprising the docking complex hold them close enough to the membrane to permit fusion (Scheller, 1995; Lin and Scheller, 1997). Predocked vesicles are thought to account for the first and fastest bout of exocytosis (Stevens and Tsujimoto, 1995; Mennerick and Matthews, 1996; von Gersdorff et al., 1996; Schikorski and Stevens, 1997) and are postulated to comprise at least a subset of the vesicles that are observed touching the membrane in electron micrographs.

In the space between the SB and the plasma membrane we observed numerous morphologically docked vesicles that were obscured by the presynaptic densities in previous TEM images of frog saccular hair cells but that have been reported in other species (Gleisner et al., 1973; Hama and Saito, 1977; Hama, 1980). Using a narrow definition of docking (no resolvable space between the vesicle and plasma membrane) similar to that used by Parsons et al. (1995) and Schikorski and Stevens (1997), we estimate that there are 32 docked vesicles per SB. A more liberal criterion (Schweizer et al., 1998) would have included additional vesicles that are present in this space but that are unlikely to be immediately available for release. The number of docked vesicles per SB is similar to that reported for ribbon synapses in retinal bipolar terminals (22 docked vesicles per ribbon; von Gersdorff et al., 1996) and larger than reported in hippocampal neurons (Schikorski and Stevens, 1997) by a factor of three, attributable primarily to the larger size of the active zones in hair cells.

Using the mean vesicle diameter measured in this study ( 34.3 $\mathrm{nm}$, measured at the middle of the membrane thickness) and assuming a specific membrane capacitance of $1 \mu \mathrm{F} / \mathrm{cm}^{2}$, we estimate a single-vesicle capacitance of $37 \mathrm{aF}$, slightly larger than previously reported (32 aF; Parsons et al., 1994). Fusion of the 32 docked vesicles at each of the 20 active zones of the hair cell (Roberts et al., 1990) thus would increase the membrane capacitance by $24 \mathrm{fF}$, similar to the magnitude of the "ultrafast" capacitance increase (30 fF) that Mennerick and Matthews (1996) hypothesize to be attributable to the rapid fusion of docked vesicles in retinal bipolar cells. An ultrafast pool has not been demonstrated in hair cells but would not have been resolved in previous capacitance measurements (Parsons et al., 1994).

The reported maximal rate of capacitance increase $(322 \mathrm{fF} / \mathrm{sec}$; Parsons et al., 1994) corresponds to 440 vesicles/sec at each SB, similar to the rate reported for bipolar neurons (500 vesicles/sec; von Gersdorff et al., 1996). At this rate the pool of docked vesicles could support only $\sim 70 \mathrm{msec}$ of exocytosis, which continues unabated for $\geq 2 \mathrm{sec}$, suggesting that release sites can be reloaded rapidly during sustained release. If the number of docked vesicles equals the number of release sites, then the turnover rate for individual release sites is 14 vesicles/sec, similar to the values reported in retinal bipolar neurons ( 23 vesicles/sec; von Gersdorff et al., 1996) and at a conventional synapse (22 vesicles/sec; Borges et al., 1995), and suggesting that ribbon-class synapses maintain high rates of exocytosis by having a large number of release sites per synapse rather than a high turnover rate at each site.

The role of the SB in accumulating and/or transporting vesicles to the active zone has been the subject of speculation for many years (Bunt, 1971; Gray and Pease, 1971) (for review, see Vollrath and Spiwoks-Becker, 1996; Wagner, 1997). In bipolar cell terminals, depolarization evokes a maximum capacitance increase ( $\sim 150 \mathrm{fF}$; von Gersdorff and Matthews, 1994) that corresponds to the membrane area of all ribbon-associated vesicles in the terminal (110 vesicles per ribbon; von Gersdorff et al., 1996), leading to the hypothesis that the ribbon-associated vesicles comprise the rapidly released pool. In frog saccular hair cells the capacitance continues to rise for a longer duration ( $\geq 2 \mathrm{sec}$ as compared with $200 \mathrm{msec}$ in bipolar terminals) and attains a higher level $(>600$ $\mathrm{fF}$ ), corresponding to a rapidly released pool of $>800$ vesicles per synapse. The $\sim 380$ SB-associated vesicles per SB can account for only the first $900 \mathrm{msec}$ of exocytosis at the maximal rate, suggesting that either reloading of the SB-associated pool is not rate limiting or the rapidly released pool contains vesicles that are not SB-associated. The inability to deplete SB-associated vesicles by $\mathrm{K}^{+}$depolarization supports the conclusion that refilling of the 
SB-associated pool is not a rate-limiting step during continuous exocytosis in these cells.

In lysed cells the SBs remained attached to the plasma membrane, and vesicles remained bound to the SB after other cytoplasmic components had been washed away. Resistance of the attachments between the SB and plasma membrane to high $\mathrm{Ca}^{2+}$ is expected, given that presynaptic $\mathrm{Ca}^{2+}$ can exceed $100 \mu \mathrm{M}$ during the normal activity of the cell (Roberts, 1993, 1994). It has been proposed that the elevated $\mathrm{Ca}^{2+}$ concentrations provided by the activation of voltage-gated $\mathrm{Ca}$ channels mobilize vesicles on the synaptic ribbons in bipolar neurons (Mennerick and Matthews, 1996). The observation that vesicles remain bound to the $\mathrm{SB}$ in $4 \mathrm{~mm} \mathrm{Ca}^{2+}$ supports mechanisms in which vesicles move without detaching.

Despite making different numbers of synapses, hair cells and bipolar terminals maintain a similar pool of cytoplasmic vesicles per synapse (10,000-30,000 in hair cells; 10,700-14,000 in bipolar terminals; von Gersdorff et al., 1996). If synapses can draw from this pool, there are enough vesicles around each synapse to account for at least several seconds of exocytosis. One second of release at the maximal rate would deplete all SB-associated vesicles plus all cytoplasmic vesicles within $95 \mathrm{~nm}$ of the SB; 2 sec would deplete the cytoplasm within $250 \mathrm{~nm}$ of the SB. The entire population of small clear-core vesicles of the cell could sustain exocytosis at the maximal rate for 20-60 sec without replenishment.

We observed omega profiles within active zones and other evidence of ongoing membrane recycling at most of the reconstructed synapses in unstimulated cells. These findings are consistent with physiological measurements showing partial activation of the Ca current (Hudspeth and Lewis, 1988) and synaptic transmission (Ishii et al., 1971) at rest. The presence of coated pits and vesicles that take up extracellular microperoxidase is strong evidence for local endocytosis at each active zone, which may contribute to the brief repriming time ( $<60 \mathrm{sec})$ measured at bipolar cell ribbon synapses (Lagnado et al., 1996) and to the local accumulation of vesicles. Similar experiments have shown that extracellular horseradish peroxidase labels ribbon-associated vesicles in photoreceptors (Schacher et al., 1974, 1976; Schaeffer and Raviola, 1978; Evans et al., 1981; Cooper and McLaughlin, 1983) and hair cells (Siegel and Brownell, 1986), suggesting that these vesicles are in the endocytic pathway.

Although there appears to be a higher concentration of endocytic sites near the active zone than far away, mapped here by electron tomography, or in other hair cells by freeze-fracture (Hama, 1980; Jacobs and Hudspeth, 1990; Roberts et al., 1990) or TEM (Gleisner et al., 1973; Hama and Saito, 1977), endocytosis also occurred far from the synapse. We found microperoxidaselabeled coated vesicles near the membrane micrometers away from synapses (by TEM), and others have observed coated pits in these regions as well (Nadol, 1990).

In these reconstructions of frog saccular hair cell synaptic regions we did not observe evidence for interconnections between intracellular tubular networks and the plasmalemma, although such connections have been reported in goldfish sacculus (Hama and Saito, 1977). A large tubular invagination of the plasma membrane was present at one of the eight synaptic reconstructions (see Fig. 9C), but no coated pits like those seen in goldfish hair cells were observed on its surface. The structure could be analogous to the large membrane invaginations that form in photoreceptors in a light-dependent manner (Cooper and McLaughlin, 1982).
Miniature postsynaptic potentials (minis), which are thought to correspond to the exocytosis of single vesicles, vary in amplitude at all synapses in which they have been observed, including hair cell afferent junctions (Ishii et al., 1971; Furukawa et al., 1978; Rossi et al., 1994). Although the source of mini variance has been debated (for review, see Bekkers, 1994; Frerking and Wilson, 1996), several studies argue that it can be intrinsic to single release sites (Bekkers et al., 1990; Frerking et al., 1995; Liu and Tsien, 1995) and could be explained by variations in vesicle size. We found a significantly narrower distribution of vesicle diameters $(C V=8.8 \pm 0.6 \%$; mean $\pm \mathrm{SEM})$ than previously reported at other synapses (e.g., 11\% $C V$ for hippocampal neurons; Bekkers et al., 1990), including ribbon synapses (De Robertis and Franchi, 1956; Lagnado et al., 1996; von Gersdorff et al., 1996), but it is unclear how much of this difference is attributable to our correcting for tracing variance and not to pooling data from different synapses. When compared with the $12 \% \mathrm{CV}$ predicted from quantal variation at single amacrine synapses (Frerking et al., 1995), our data indicate that either hair cell synapses have less quantal variation than amacrine cell synapses or factors in addition to vesicle volume contribute to quantal variation.

The ultrastructural differences between afferent synapses in hair cells and bipolar neurons may reflect functional differences between synapses specialized for sustained or transient release (but for an alternative view, see Lagnado et al., 1996). The threeto fourfold greater number of vesicles on each SB may play a role in the ability of hair cells to maintain continuous exocytosis for $>10$ times longer than bipolar neurons (Parsons et al., 1994; von Gersdorff and Matthews, 1994) but is insufficient by itself to account for the difference. One possibility, which also accounts for our inability to deplete vesicles on the $\mathrm{SB}$, is that the refilling of the ribbon-associated pool is rate limiting in bipolar neurons, whereas the reloading of release sites is rate limiting in hair cells. The difference does not appear to be at the release sites, which have similar turnover rates in the two cell types, but could be explained by a faster refilling of the SB-associated pool in hair cells. The larger surface area of SBs in hair cells would favor faster refilling of this pool, but it is not clear that any such simple explanation will prove adequate. Additional experiments will be required to resolve this issue.

\section{REFERENCES}

Armstrong CE, Roberts WM (1998) Electrical properties of frog saccular hair cells: distortion by enzymatic dissociation. J Neurosci 18:2962-2973.

Bashein G, Detmer PR (1994) Centroid of a polygon. In: Graphics gems IV (Heckbert PS, ed), pp 3-6. New York: Academic.

Bekkers JM (1994) Quantal analysis of synaptic transmission in the central nervous system. Curr Opin Neurobiol 4:360-365.

Bekkers JM, Richerson GB, Stevens CF (1990) Origin of variability in quantal size in cultured hippocampal neurons and hippocampal slices. Proc Natl Acad Sci USA 87:5359-5362.

Borges S, Gleason E, Turelli M, Wilson M (1995) The kinetics of quantal transmitter release from retinal amacrine cells. Proc Natl Acad Sci USA 92:6896-6900.

Bunt AH (1971) Enzymatic digestion of synaptic ribbons in amphibian retinal photoreceptors. Brain Res 25:571-577.

Cooper NGF, McLaughlin BJ (1982) Structural correlates of physiological activity in chick photoreceptor synaptic terminals: effect of light and dark stimulation. J Ultrastruct Res 79:58-73.

Cooper NGF, McLaughlin BJ (1983) Tracer uptake by photoreceptor synaptic terminals. J Ultrastruct Res 84:252-267.

De Robertis E, Franchi CM (1956) Electron microscope observations on synaptic vesicles in synapses of the retinal rods and cones. J Biophys Biochem Cytol 2:307-317.

Evans JA, Liscum L, Hood DC, Holtzman E (1981) Uptake of horse- 
radish peroxidase by presynaptic terminals of bipolar cells and photoreceptors of the frog retina. J Histochem Cytochem 29:511-518.

Frerking M, Wilson M (1996) Saturation of postsynaptic receptors at central synapses? Curr Opin Neurobiol 6:395-403.

Frerking M, Borges S, Wilson M (1995) Variation in GABA mini amplitude is the consequence of variation in transmitter concentration. Neuron 15:885-895.

Fry KR, Spira AW (1980) An ethanolic phosphotungstic acid (EPTA) analysis of photoreceptor and synaptic ultrastructure in the guinea pig retina. J Histochem Cytochem 28:141-148.

Furukawa T, Hayashida Y, Matsuura S (1978) Quantal analysis of the size of excitatory postsynaptic potentials at synapses between hair cells and afferent nerve fibres in goldfish. J Physiol (Lond) 276:211-226.

Gleisner L, Flock A, Wersäll J (1973) The ultrastructure of the afferent synapse on hair cells in the frog labyrinth. Acta Otolaryngol (Stockh) 76:199-207.

Gray EG, Pease HL (1971) On understanding the organisation of the retinal receptor synapses. Brain Res 35:1-15.

Hama K (1980) Fine structure of the afferent synapse and gap junctions on the sensory hair cell in the saccular macula of goldfish: a freezefracture study. J Neurocytol 9:845-860.

Hama K, Saito K (1977) Fine structure of the afferent synapse of the hair cells in the saccular macula of the goldfish, with special reference to the anastomosing tubules. J Neurocytol 6:361-373.

Heidelberger R, Heinemann C, Neher E, Matthews G (1994) Calcium dependence of the rate of exocytosis in a synaptic terminal. Nature 371:513-515.

Hessler D, Young SJ, Carragher BO, Martone M, Lamont S, Whittaker M, Milligan RA, Masliah E, Hinshaw JE, Ellisman MH (1992) SYNU: software for visualization of three-dimensional biological structures. NeuroImage 1:55-67.

Hudspeth AJ, Issa NP (1996) Confocal-microscopic visualization of membrane addition during synaptic exocytosis at presynaptic active zones of hair cells. Cold Spring Harbor Symp Quant Biol 61:303-307.

Hudspeth AJ, Lewis RS (1988) Kinetic analysis of voltage- and iondependent conductances in saccular hair cells of the bull frog, Rana catesbeiana. J Physiol (Lond) 400:237-274.

Ishii Y, Matsuura S, Furukawa T (1971) Quantal nature of transmission at the synapse between hair cells and eighth nerve fibers. Jpn J Physiol 21:779-789.

Issa NP, Hudspeth AJ (1994) Clustering of $\mathrm{Ca}^{2+}$ channels and $\mathrm{Ca}^{2+}$ activated $\mathrm{K}^{+}$channels at fluorescently labeled presynaptic active zones of hair cells. Proc Natl Acad Sci USA 91:7578-7582.

Jacobs RA, Hudspeth AJ (1990) Ultrastructural correlates of mechanoelectrical transduction in hair cells of the bullfrog's internal ear. Cold Spring Harbor Symp Quant Biol 55:547-561.

Jing Z, Sachs F (1991) Alignment of tomographic projections using an incomplete set of fiducial markers. Ultramicroscopy 35:37-43.

Lagnado L, Gomis A, Job C (1996) Continuous vesicle cycling in the synaptic terminal of retinal bipolar cells. Neuron 17:957-967.

Leake PA, Snyder RL (1987) Uptake of horseradish peroxidase from perilymph by cochlear hair cells. Hear Res 22:153-171.

Lenzi D, Roberts WM (1994) Calcium signaling in hair cells: multiple roles in a compact cell. Curr Opin Neurobiol 4:496-502.

Lin RC, Scheller RH (1997) Structural organization of the synaptic exocytosis core complex. Neuron 19:1087-1094.

Liu G, Tsien RW (1995) Properties of synaptic transmission at single hippocampal synaptic boutons. Nature 375:404-408.

Luther PK (1992) Sample shrinkage and radiation damage. In: Electron tomography: three-dimensional imaging with the transmission electron microscope (Frank J, ed), pp 39-60. New York: Plenum.

Martinez-Dunst C, Michaels RL, Fuchs PA (1997) Release sites and calcium channels in hair cells of the chick's cochlea. J Neurosci 17:9133-9144.

Mellman I (1996) Endocytosis and molecular sorting. Annu Rev Cell Dev Biol 12:575-625.

Mennerick S, Matthews G (1996) Ultrafast exocytosis elicited by calcium current in synaptic terminals or retinal bipolar neurons. Neuron 17:1241-1249.

Miller MR, Beck J (1987) Heterotopic synaptic bodies in the auditory hair cells of adult lizards. Anat Rec 218:338-344.

Nadol JB (1990) Synaptic morphology of inner and outer hair cells of the human organ of Corti. J Electron Microsc Tech 15:187-196.

Neher E (1998) Vesicle pools and $\mathrm{Ca}^{2+}$ microdomains: new tools for understanding their roles in neurotransmitter release. Neuron 20:389-399.

Parsons TD, Lenzi D, Almers W, Roberts WM (1994) Calciumtriggered exocytosis and endocytosis in an isolated presynaptic cell capacitance measurements in saccular hair cells. Neuron 13:875-883.

Parsons TD, Coorsen JR, Horstmann H, Almers W (1995) Docked granules, the exocytic burst, and the need for ATP hydrolysis in endocrine cells. Neuron 15:1085-1096.

Radermacher M (1992) Weighted backprojection methods. In: Electron tomography: three-dimensional imaging with the transmission electron microscope (Frank J, ed), pp 91-115. New York: Plenum.

Rao-Mirotznik R, Harkins AB, Buchsbaum G, Sterling P (1995) Mammalian rod terminal: architecture of a binary synapse. Neuron 14:561-569.

Rieke F, Schwartz EA (1994) A cGMP-gated current can control exocytosis at cone synapses. Neuron 13:863-873.

Rieke F, Schwartz EA (1996) Asynchronous transmitter release: control of exocytosis and endocytosis at the salamander rod synapse. J Physiol (Lond) 493:1-8.

Roberts WM (1993) Spatial calcium buffering in saccular hair cells. Nature 363:74-76.

Roberts WM (1994) Localization of calcium signals by a mobile calcium buffer in frog saccular hair cells. J Neurosci 14:3246-3262.

Roberts WM, Jacobs RA, Hudspeth AJ (1990) Colocalization of ion channels involved in frequency selectivity and synaptic transmission at presynaptic active zones of hair cells. J Neurosci 10:3664-3684.

Roberts WM, Jacobs RA, Hudspeth AJ (1991) The hair cell as a presynaptic terminal. Ann NY Acad Sci 635:221-233.

Rossi ML, Martini M, Pelucchi B, Fesce R (1994) Quantal nature of synaptic transmission at the cytoneural junction in the frog labyrinth. J Physiol (Lond) 478:17-35.

Sato T (1967) A modified method for lead staining. J Electron Microsc 16:133-141.

Schacher SM, Holtzman E, Hood DC (1974) Uptake of horseradish peroxidase by frog photoreceptor synapses in the dark and the light. Nature 249:261-263.

Schacher SM, Holtzman E, Hood DC (1976) Synaptic activity of frog retinal photoreceptors. A peroxidase uptake study. J Cell Biol 70:178-192.

Schaeffer SF, Raviola E (1978) Membrane recycling in the cone cell endings of the turtle retina. J Cell Biol 79:802-825.

Scheller RH (1995) Membrane trafficking in the presynaptic nerve terminal. Neuron 14:893-897.

Schikorski T, Stevens CF (1997) Quantitative ultrastructural analysis of hippocampal excitatory synapses. J Neurosci 17:5858-5867.

Schroeter JP, Bretaudiere JP (1996) SUPRIM: easily modified image processing software. J Struct Biol 116:131-137.

Schweizer FE, Dresbach T, DeBello WM, O’Connor V, Augustine GJ, Betz H (1998) Regulation of neurotransmitter release kinetics by NSF. Science 279:1203-1206.

Siegel JH, Browell WE (1986) Synaptic and Golgi membrane recycling in cochlear hair cells. J Neurocytol 15:311-328.

Smith JE, Reese TS (1980) Use of aldehyde fixatives to determine the rate of synaptic transmitter release. J Exp Biol 89:19-29.

Soto GE, Young SJ, Martone ME, Deerinck TJ, Lamont S, Carragher BO, Hama K, Ellisman MH (1994) Serial section tomography: a method for three-dimensional reconstruction of large structures. NeuroImage 1:230-243.

Stevens CF, Tsujimoto T (1995) Estimates for the pool size of releasable quanta at a single synapse for the time required to refill the pool. Proc Natl Acad Sci USA 92:846-849.

Usukura J, Yamada E (1987) Ultrastructure of the synaptic ribbons in photoreceptor cells of Rana catesbeiana revealed by freeze-etching and freeze-substitution. Cell Tissue Res 247:483-488.

Vollrath L, Spiwoks-Becker I (1996) Plasticity of retinal ribbon synapses. Microsc Res Tech 35:472-487.

von Gersdorff H, Matthews G (1994) Dynamics of synaptic vesicle fusion and membrane retrieval in synaptic terminals. Nature 367:735-739.

von Gersdorff H, Vardi E, Matthews G, Sterling P (1996) Evidence that vesicles on the synaptic ribbon of retinal bipolar neurons can be rapidly released. Neuron 16:1221-1227.

Wagner HJ (1997) Presynaptic bodies ("ribbons"): from ultrastructural observations to molecular perspectives. Cell Tissue Res 287:435-446.

Young SJ, Fan GY, Hessler D, Lamont S, Elvins TT, Hadida M, Hanyzewski G, Durkin JW, Hubbard P, Kindalman G, Wong E, Greenberg D, Karin S, Ellisman MH (1996) Implementing a collaboratory for digital microscopy. Int J Supercomput Appl 10:170-181. 